Acta Crystallographica Section C

Crystal Structure

Communications

ISSN 0108-2701

\section{A study of the pyramidality index in tris(2,4,6-triisopropylphenyl)phos- phonium perchlorate}

\author{
René T. Boeré* and Yuankui Zhang
}

Department of Chemistry and Biochemistry, University of Lethbridge, Lethbridge AB, Canada T1K $3 \mathrm{M} 4$

Correspondence e-mail: boere@uleth.ca

Received 9 July 2013

Accepted 24 July 2013

The title compound, $\mathrm{C}_{45} \mathrm{H}_{70} \mathrm{P}^{+} \cdot \mathrm{ClO}_{4}{ }^{-}$or $\left[\right.$Tripp $\left.3 \mathrm{PH}^{+}\right]\left[\mathrm{ClO}_{4}{ }^{-}\right]$, was produced from the perchlorate salt of the corresponding radical cation Tripp $_{3} \mathrm{P}^{++}$through very slow $\mathrm{H}$-atom abstraction reactions in a solution of 1,2-dimethoxyethane (Tripp is 2,4,6triisopropylphenyl). The $\mathrm{H}$ atom on the $\mathrm{P}$ atom was located in a difference map and was freely refined with an isotropic displacement consistent with full occupancy $[\mathrm{P}-\mathrm{H}=$ 1.37 (3) $\AA$ ]. It is the most sterically congested triarylphosphonium salt yet to be reported and has a 'propeller' arrangement of the three Tripp groups around the $\mathrm{P}$ atom, with the protonation site located along the molecular threefold axis. There are short contacts between the flanking isopropyl methine $\mathrm{H}$ atoms and the $\mathrm{P}$ atom $[\mathrm{P} \cdots \mathrm{H}=1.99(2)-$ 2.17 (2) $\AA$ ]. The sum of the angles around the $\mathrm{P}$ atom $\left[\Sigma(\mathrm{C}-\mathrm{P}-\mathrm{C})=349.9(6)^{\circ}\right]$ is a convenient index of pyramidality for tricoordinate centres. This value is significantly larger than in analogous $\mathrm{Mes}_{3} \mathrm{PH}^{+}$salts (Mes is mesityl or 2,4,6trimethylphenyl), for which the average of the three reported structures in the literature is $\Sigma(\mathrm{C}-\mathrm{P}-\mathrm{C})=345.3(6)^{\circ}$. For comparison, in the ubiquitous $\mathrm{Ph}_{3} \mathrm{PH}^{+}$salts, this parameter has a typical average value of only $333.3(9)^{\circ}$. The value of $\Sigma(\mathrm{C}-$ $\mathrm{P}-\mathrm{C})$ in the title compound is midway between that of the neutral phosphane $\operatorname{Tripp}_{3} \mathrm{P}\left[334.4(6)^{\circ}\right]$ and the phosphoniumyl radical cation $\operatorname{Tripp}_{3} \mathrm{P}^{++}\left[359.8(2)^{\circ}\right]$. This geometrical feature provides additional support for the assignment as a phosphonium salt.

Keywords: crystal structure; triarylphosphonium ions; pyramidality index; phosphoniumyl radical cations.

\section{Introduction}

There is intense current interest in the preparation and isolation of stable phosphoniumyl radical cations (Bullock et al., 2013; Boeré et al., 2008; Sasaki et al., 2002, 2004, 2006; Sasaki \& Yoshifuji, 2007; Chalier et al., 1996). Exhaustive studies have shown that this can be achieved only when very bulky substituents are employed, and thus far all the successful examples use bulky aryl groups. The widely used 2,4,6-trimethylphenyl (Mes) group has been shown to lead to persistent radical cations of the type $\left[\mathrm{Mes}_{3} \mathrm{P}^{++}\right]$, but these are still reactive species and salts have never been isolated. By increasing the steric bulk through the use of various aryl groups that have in common flanking 2,6-diisopropyl groups, designated ${ }^{\mathrm{i}-\mathrm{Pr}_{2}} \mathrm{Ar}$, a number of stable $\left[{ }^{\mathrm{i}-\mathrm{Pr}_{2}} \mathrm{Ar}_{3} \mathrm{P}^{++}\right]$radical cations have now been reported. Significantly, Pan et al. (2013) recently reported the first crystal structures in this class with a number of salts containing the 2,4,6-triisopropylphenyl (Tripp) group. Three structures of the type $\left[\operatorname{Tripp}_{3} \mathrm{P}^{++}\right] X^{-}$were reported with $X^{-}=\mathrm{SbF}_{6}{ }^{-},\left[\mathrm{Al}\left\{\mathrm{OC}\left(\mathrm{CF}_{3}\right)_{3}\right\}_{4}{ }^{-}\right]$and [Al $\{\mathrm{OCMe}-$ $\left.\left.\left(\mathrm{CF}_{3}\right)_{2}\right\}_{4}{ }^{-}\right]$, as well as $\left[\mathrm{MesTripp}{ }_{2} \mathrm{P}^{+}\right]\left[\mathrm{Al}\left\{\mathrm{OC}\left(\mathrm{CF}_{3}\right)_{3}\right\}_{4}{ }^{-}\right]$. Each of the $\left[\right.$ Tripp $\left._{3} \mathrm{P}^{++}\right] X^{-}$salts was found to have structures that are planar within experimental error, with an average of the sums of the angles around the $\mathrm{P}$ atom (for background on this parameter, see: Boeré \& Zhang, 2005), denoted $\Sigma(\mathrm{C}-\mathrm{P}-\mathrm{C})$, of $359.8(2)^{\circ}$, whilst the [MesTripp ${ }_{2} \mathrm{P}^{+}$] cation was found to be mildly pyramidal, with a $\Sigma(\mathrm{C}-\mathrm{P}-\mathrm{C})$ value of $349.15(6)^{\circ}$ (Pan et al., 2013). These workers report that solutions of $\left[\right.$ Tripp $\left.{ }_{3} \mathrm{P}^{+}\right] X^{-}$salts retain their colour when reacted with the $\mathrm{H}$-atom source ${ }^{n} \mathrm{Bu}_{3} \mathrm{SnH}$. We have also prepared $\left[\operatorname{Tripp}_{3} \mathrm{P}^{++}\right] X^{-}$salts with a variety of anions via the corresponding silver(I) salts with the identity of the radical cations confirmed by electron paramagnetic resonance (EPR) spectroscopy (see below). Although we obtained stable solid materials with $X^{-}=\mathrm{SbF}_{6}{ }^{-}, \mathrm{AsF}_{6}{ }^{-}$and $\mathrm{ClO}_{4}{ }^{-}$, we have been unable to obtain diffraction-quality crystals. However, solutions of $\left[\operatorname{Tripp}_{3} \mathrm{P}^{+}\right]\left[\mathrm{ClO}_{4}{ }^{-}\right]$slowly deposit large blocks over

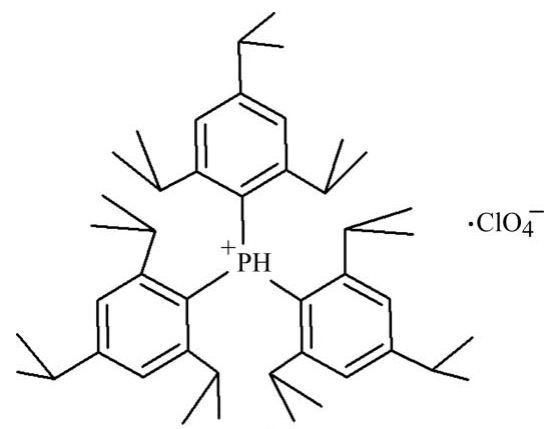

(I)

long periods of storage in the dark that, while streaked with residual red colour (presumably from occluded radical cation), are predominantly colourless. The structure determination reported here demonstrates that these are the diamagnetic phosphonium salts which are probably obtained by very slow $\mathrm{H}$-atom abstraction reactions. Thus, our results do not contradict the reports that a rapid reaction with reactive $\mathrm{H}$ atom sources such as ${ }^{n} \mathrm{Bu}_{3} \mathrm{SnH}$ does not occur for the [Tripp $\mathrm{P}_{3} \mathrm{P}^{+}$] radical cation; over time, perhaps with lattice forces as a driving mechanism, the $\mathrm{H}$-atom abstraction product does form even from relatively unreactive $\mathrm{H}$-atom sources (other molecules of the radical cation or the 1,2-dimethoxyethane solvent). Radical attack on $\mathrm{C}-\mathrm{H}$ bonds by phosponiumyl ions has recently been reported and can be 
made catalytic with the addition of suitable Lewis acids (Ménard et al., 2013).

\section{Experimental}

\subsection{Synthesis and crystallization}

A small quantity of tris(2,4,6-triisopropylphenyl)phosphane $\left(\operatorname{Tripp}_{3} \mathrm{P}\right)$, prepared according to the literature method of Sasaki et al. (2002), was placed in the wide limb [outer diameter (od) $8 \mathrm{~mm}$ ] end of a Pyrex ' $\mathrm{T}$ ' tube, and a grain of $\mathrm{AgClO}_{4}$ was added to the solid. Dried degassed 1,2-dimethoxyethane (DME; distilled under $\mathrm{N}_{2}$ from molten sodium) was added by syringe $(1.0 \mathrm{ml})$ and the reaction mixture was immediately connected to a vacuum line and freeze-thaw degassed three times. While still under vacuum, the opening was sealed by melting the glass. After cooling the hot glass, the reaction mixture was carefully thawed and shaken to dissolve the reagents. An immediate intense deep-red colour developed. Small quantities of the red material were decanted into the other arm of the vessel, which consists of a $4 \mathrm{~mm}$ od EPR tube. Solvent was then carefully distilled into the narrow limb to ensure a dilute solution of the radical. The EPR spectrum (X-band, 9.7674 GHz) was determined (Fig. 1) at ambient temperature ( $c a 291 \mathrm{~K})$. The spectrum displays the characteristic doublet from coupling to the single ${ }^{31} \mathrm{P}$ nucleus with a hyperfine splitting of $23.62 \mathrm{mT}$ and a third-order corrected $g$ value of 2.0060. Unresolved coupling to $\mathrm{H}$ atoms of the 2,4,6triisopropylphenyl ring is evident from the need to include about $15 \%$ Gaussian character to the simulated line shape. The spectrum displays unsymmetrical linewidths, which fit to a 'slow tumbling' equation: $\mathrm{LW}=(0.650-0.10 \mathrm{~m}) \mathrm{mT}$ (Murphy, 2009). This data may be compared to a reported $a\left({ }^{31} \mathrm{P}\right)=$ $23.3 \mathrm{mT}$ and (likely uncorrected) $g=2.008$ for solutions made up from the corresponding $\mathrm{SbF}_{6}{ }^{-}$salt in $\mathrm{CH}_{2} \mathrm{Cl}_{2}$ (Pan et al.,

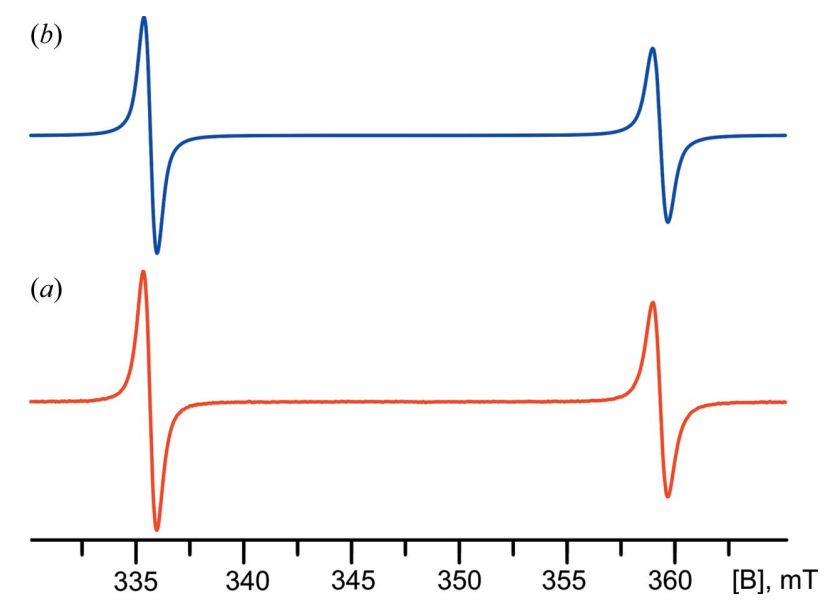

Figure 1

(a) The X-band $(9.8 \mathrm{GHz})$ EPR spectrum of a solution of $\left[\operatorname{Tripp}_{3} \mathrm{P}^{++}\right]$$\left[\mathrm{ClO}_{4}{ }^{-}\right]$in 1,2-dimethoxyethane at $291 \mathrm{~K}$, showing the expected doublet with $a\left({ }^{31} \mathrm{P}\right)=23.62 \mathrm{mT}$. (b) The simulation obtained in which the linewidths fit to $(0.650-0.10 \mathrm{~m}) \mathrm{mT}$, consistent with incomplete averaging of the $g$ and $a$ tensors caused by slow tumbling in solution.
Table 1

Experimental details.

\begin{tabular}{|c|c|}
\hline \multicolumn{2}{|l|}{ Crystal data } \\
\hline Chemical formula & $\mathrm{C}_{45} \mathrm{H}_{70} \mathrm{P}^{+} \cdot \mathrm{ClO}_{4}^{-}$ \\
\hline$M_{\mathrm{r}}$ & 741.43 \\
\hline Crystal system, space group & Orthorhombic, $P 2_{1} 2_{1} 2_{1}$ \\
\hline Temperature (K) & 173 \\
\hline$a, b, c(\AA)$ & $13.6822(14), 17.0621(18), 18.660$ (2) \\
\hline$V\left(\AA^{3}\right)$ & $4356.1(8)$ \\
\hline$Z$ & 4 \\
\hline Radiation type & Mo $K \alpha$ \\
\hline$\mu\left(\mathrm{mm}^{-1}\right)$ & 0.16 \\
\hline Crystal size $(\mathrm{mm})$ & $0.38 \times 0.21 \times 0.17$ \\
\hline \multicolumn{2}{|l|}{ Data collection } \\
\hline Diffractometer & $\begin{array}{l}\text { Bruker APEXII CCD area-detector } \\
\text { diffractometer }\end{array}$ \\
\hline Absorption correction & Multi-scan (SADABS; Bruker, 2008) \\
\hline$T_{\min }, T_{\max }$ & $0.941,0.973$ \\
\hline $\begin{array}{l}\text { No. of measured, independent and } \\
\quad \text { observed }[I>2 \sigma(I)] \text { reflections }\end{array}$ & $61837,10021,7663$ \\
\hline$R_{\text {int }}$ & 0.054 \\
\hline$(\sin \theta / \lambda)_{\max }\left(\AA^{-1}\right)$ & 0.650 \\
\hline \multicolumn{2}{|l|}{ Refinement } \\
\hline$R\left[F^{2}>2 \sigma\left(F^{2}\right)\right], w R\left(F^{2}\right), S$ & $0.039,0.097,1.02$ \\
\hline No. of reflections & 10021 \\
\hline No. of parameters & 483 \\
\hline No. of restraints & 0 \\
\hline H-atom treatment & $\begin{array}{l}\mathrm{H} \text { atoms treated by a mixture of } \\
\text { independent and constrained } \\
\text { refinement }\end{array}$ \\
\hline$\Delta \rho_{\max }, \Delta \rho_{\min }\left(\mathrm{e} \AA^{-3}\right)$ & $0.35,-0.31$ \\
\hline Absolute structure & Refined as an inversion twin \\
\hline Absolute structure parameter & $0.49(7)$ \\
\hline
\end{tabular}

2013). Repeated attempts to grow crystals of such salts with a variety of anions from many different solvents were unsuccessful, but over a very long time (4-5 years) during storage of the sealed T-tube in the dark at ambient temperature, large blocks developed containing red tinted streaks, but which are nevertheless predominantly colourless. Successful solution of the single-crystal structure shows that rather than the expected radical cation salt $\left[\operatorname{Tripp}_{3} \mathrm{P}^{++}\right]\left[\mathrm{ClO}_{4}{ }^{-}\right]$, which has a deep-red colour, the crystals are composed of the colourless diamagnetic phosphonium salt, $\left[\operatorname{Tripp}_{3} \mathrm{PH}^{+}\right]\left[\mathrm{ClO}_{4}{ }^{-}\right]$, (I). We believe that this product results from slow $\mathrm{H}$-atom abstraction, either from the reaction medium (solvent or adventitious moisture) or from other cation molecules. The other possibility, that excess silver salt has hydrolysed to $\mathrm{HClO}_{4}$ which then protonated unreacted phosphane, cannot be ruled out entirely, but does not seem to be consistent with the timescale of the reaction that was established from monitoring the EPR spectra.

\subsection{Refinement}

Crystal data, data collection and structure refinement details are summarized in Table $1 . \mathrm{H}$ atoms attached to $\mathrm{C}$ atoms were treated as riding, with $\mathrm{C}-\mathrm{H}=0.98 \AA$ and $U_{\text {iso }}(\mathrm{H})=1.5 U_{\text {eq }}(\mathrm{C})$ for methyl, $\mathrm{C}-\mathrm{H}=1.00 \AA$ and $U_{\text {iso }}(\mathrm{H})=$ $1.2 U_{\text {eq }}(\mathrm{C})$ for methine, and $\mathrm{C}-\mathrm{H}=0.95 \AA$ and $U_{\text {iso }}(\mathrm{H})=$ $1.2 U_{\text {eq }}(\mathrm{C})$ for aromatic $\mathrm{H}$ atoms. The phosphonium $\mathrm{H}$ atom 
Table 2

Pyramidality index in triarylphosphonium ion structures.

\begin{tabular}{llll}
\hline Aryl group & CSD refcode & $\Sigma(\mathrm{C}-\mathrm{P}-\mathrm{C})\left(^{\circ}\right)$ & Reference \\
\hline $\mathrm{Ph}_{3} \mathrm{PH}^{+}$ & ABERAA & $332.7(5)$ & Junk \& Atwood (1999) \\
$\mathrm{Ph}_{3} \mathrm{PH}^{+}$ & BARVUL & $334(3)$ & Boorman et al. $(1981)$ \\
$\mathrm{Ph}_{3} \mathrm{PH}^{+}$ & KAXQUQ & $333.0(6)$ & Hagenbach \& Abram (2005) \\
{$\left[2,4,6-(\mathrm{MeO})_{3} \mathrm{C}_{6} \mathrm{H}_{2}\right]_{3} \mathrm{PH}^{+}$} & LAFGEE10 & $338.9(5)$ & Dunbar \& Quillevere (1993) \\
{$\left[2,4,6-(\mathrm{MeO})_{3} \mathrm{C}_{6} \mathrm{H}_{2}\right]_{3} \mathrm{PH}^{+}$} & TAJGAM & $342.3(6)$ & Dunbar \& Pence (1991) \\
{$\left[2,4,6-(\mathrm{MeO})_{3} \mathrm{C}_{6} \mathrm{H}_{2}\right]_{3} \mathrm{PH}^{+}$} & WIBJEW & $337.5(9)$ & Dunbar \& Quillevere (1993) \\
{$\left[2,4,6-(\mathrm{MeO})_{3} \mathrm{C}_{6} \mathrm{H}_{2}\right]_{3} \mathrm{PH}^{+}$} & WIBJIA & $343.0(7)$ & Dunbar \& Quillevere (1993) \\
$\mathrm{Mes}_{3} \mathrm{PH}^{+}$ & LUWPOJ & $345.3(5)$ & Ménard \& Stephan (2010) \\
$\mathrm{Mes}_{3} \mathrm{PH}^{+}$ & QUHCAY & $345.6(6)$ & Jiang et al. $(2009)$ \\
$\mathrm{Mes}_{3} \mathrm{PH}^{+}$ & XALCUK & $345.1(6)$ & Schäfer et al. $(2011)$ \\
$\mathrm{Tripp}_{3} \mathrm{PH}^{+}$ & & $349.9(6)$ & This work \\
\hline
\end{tabular}

search include four exemplars of tris(2,4,6-trimethoxyphenyl)phosphonium salts [refcodes LAFGEE10, WIBJEW and WIBJIA (Dunbar \& Quillevere, 1993), and TAJGAM (Dunbar \& Pence, 1991)]. These structures show greater variation in pyramidality compared to $\mathrm{Ph}_{3} \mathrm{PH}^{+}$apparently because the substituents take differing orientations around the $\mathrm{P}$ atom, resulting in an average $\Sigma(\mathrm{C}-\mathrm{P}-\mathrm{C})$ value of $340(3)^{\circ}$, with a rather large s.u. value. The bulkiest structures reported to date contain $\mathrm{Mes}_{3} \mathrm{PH}^{+}$ions, for which three structures are present in the CSD [refcodes LUWPOJ (Ménard \& Stephan, 2010), QUHCAY (Jiang et al., 2009) and XALCUK (Schäfer et al., 2011)]. These display fairly consistent structural was located in a difference Fourier map and refined freely with an isotropic $U$ value. The 'CALC VOID' routine in PLATON (Spek, 2009) was used to identify solvent-accessible voids in the unit cell.

\section{Results and discussion}

We report here the structure determination of tris $(2,4,6-$ triisopropylphenyl)phosphonium perchlorate, $\left[\mathrm{Tripp}_{3} \mathrm{PH}^{+}\right]-$ $\left[\mathrm{ClO}_{4}^{-}\right]$, (I) (Fig. 2). There are only a relatively small number of structures reported that contain $\mathrm{Ar}_{3} \mathrm{PH}^{+}$cations. A search of the Cambridge Structural Database (CSD, Version 5.34, with updates to May 2013; Allen, 2002) resulted in 45 different structures; of these, 24 contain the $\mathrm{Ph}_{3} \mathrm{PH}^{+}$ion and another eight are closely related [such as $\left(\mathrm{XC}_{6} \mathrm{H}_{4}\right) \mathrm{Ph}_{2} \mathrm{PH}^{+}$ions]. Taking three of the more accurately determined structures containing the $\mathrm{Ph}_{3} \mathrm{PH}^{+}$ion [refcodes ABERAA (Junk \& Atwood, 1999), BARVUL (Boorman et al., 1981) and KAXQUW (Hagenbach \& Abram, 2005)] yields an average $\Sigma(\mathrm{C}-\mathrm{P}-\mathrm{C})$ value of $333.3(9)^{\circ}$ (see Table 2). Obviously more sterically bulky phosphonium salts identified from this

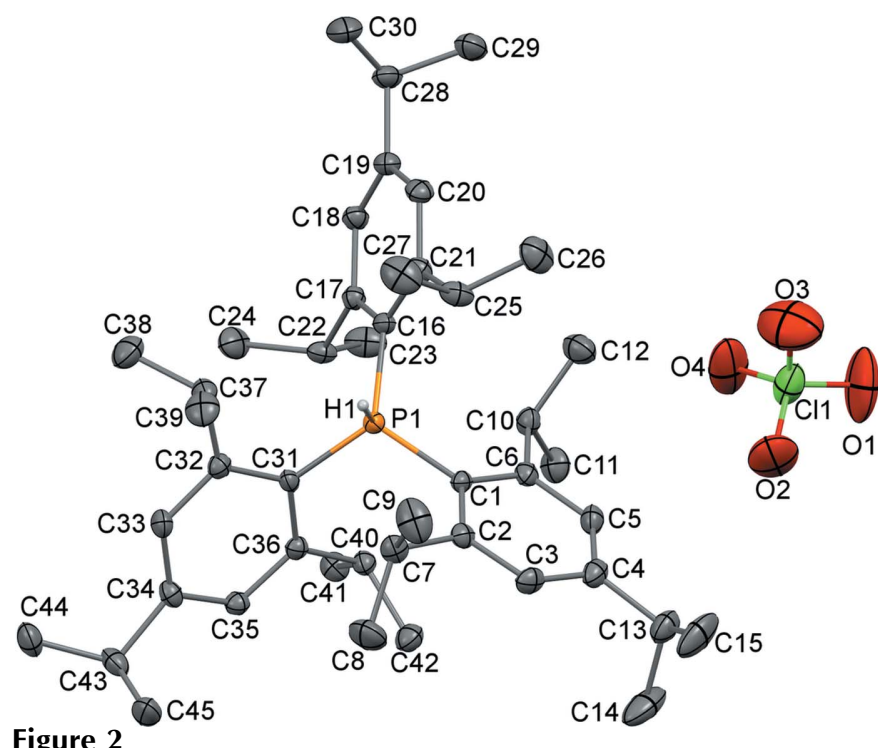

The structure of the cation-anion pair in (I), with displacement elipsoids drawn at the $40 \%$ probability level and showing the atom-numbering scheme. $\mathrm{H}$ atoms on $\mathrm{C}$ atoms have been omitted to enhance visibility. features with 'propeller' orientations of the aryl rings, yielding an average $\Sigma(\mathrm{C}-\mathrm{P}-\mathrm{C})$ value of $345.3(6)^{\circ}$. The increased steric bulk of the Tripp substituent is then clearly observable by the $\Sigma(\mathrm{C}-\mathrm{P}-\mathrm{C})$ value of $349.9(6)^{\circ}$ in (I). The pyramidality index of (I) places it firmly between the planarity observed in Tripp $_{3} \mathrm{P}^{++}$salts, with an average $\Sigma(\mathrm{C}-\mathrm{P}-\mathrm{C})$ value of $359.8(2)^{\circ}$ (Pan et al., 2013), and the more pyramidal neutral Tripp $_{3} \mathrm{P}$, for which the $\Sigma\left(\mathrm{C}-\mathrm{P}-\mathrm{C}\right.$ ) value is $334.4(6)^{\circ}$ (refcode XUNXEJ; Sasaki et al., 2002). This distinctive structural feature provides strong support for our identification of the title cation as a phosphonium ion.

An unusual feature of the structure of (I), and one that is likely a direct consequence of steric crowding, is the presence of short intramolecular distances between atom $\mathrm{H} 1$ on $\mathrm{P} 1$ and the three flanking isopropyl group methine $\mathrm{H}$ atoms $\mathrm{H} 7, \mathrm{H} 25$ and $\mathrm{H} 37$, with $\mathrm{H} \cdots \mathrm{H}$ distances ranging from 1.99 (2) to 2.17 (2) $\AA$, and thus shorter than or equal to the sums of the van der Waals radii of $2.18 \AA$. Perusal of space-filling mol-

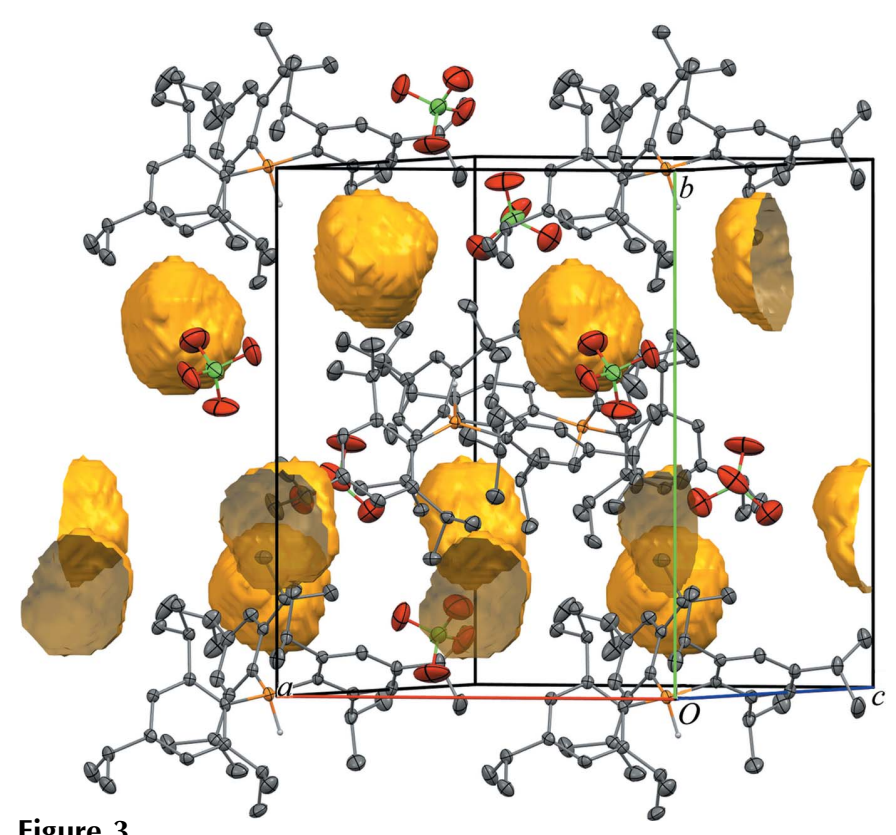

Figure 3

A packing view with the $b$ axis vertical and the bisector of $a$ and $c$ perpendicular. The contact surfaces of the small void volumes in the lattice are shown (each cavity has a volume of $30 \AA^{3}$ ). H atoms on $\mathrm{C}$ atoms have been omitted to enhance visibility. 
ecular models shows that atom H1 is completely buried amongst these flanking isopropyl group atoms. Severe steric crowding is also indicated by distortions in the aryl ring. In particular, the C31-ring shows a distinct boat conformation; from the least-squares plane defined by atoms C32/C33/C35/ C36 (r.m.s. deviation $=0.002 \AA$ ), the other atoms deviate by 0.058 (4) (C34) 0.064 (4) (C31) and 0.627 (5) § (P1). In contrast, in neutral Tripp ${ }_{3} \mathrm{P}$, the most distorted ring has the ipso-C and $\mathrm{P}$ atoms 0.046 and $0.491 \AA$, respectively, out of plane (refcode XUNXEJ; Sasaki et al., 2002). The final feature of note in this structure is the presence of four voids in the unit cell with a volume of $30 \AA^{3}$ each (Fig. 3). These were identified with the help of the 'CALC VOID' routine in PLATON (Spek, 2009), as well as with Mercury (Macrae et al., 2006). However, the voids seem to be empty of any nuclei. Most likely these cavities are merely consequences of packing such large cations with the relatively small perchlorate anions, but we cannot rule out the possibility that some contain free electrons left over from the $\mathrm{H}$-atom abstraction reactions that lead to these phosphonium salts.

The Natural Sciences and Engineering Research Council of Canada is gratefully acknowledged for Discovery Grants and an I2I award to RTB. We thank GEM Systems, Richmond Hill, Ontario, for their interest in our work on bulky phosphanes. The diffractometer was purchased with the help of NSERC and the University of Lethbridge, while the EPR spectrometer was funded by the Canada Foundation for Innovation, the Government of Alberta and Bruker Canada, Inc.

Supplementary data for this paper are available from the IUCr electronic archives (Reference: FN3145). Services for accessing these data are described at the back of the journal.

\section{References}

Allen, F. H. (2002). Acta Cryst. B58, 380-388.

Boeré, R. T., Bond, A. M., Cronin, S., Duffy, N. W., Hazendonk, P., Masuda, J. D., Pollard, K., Roemmele, T. L., Tran, P. \& Zhang, Y. (2008). New J. Chem. 32, 214-231.

Boeré, R. T. \& Zhang, Y. (2005). J. Organomet. Chem. 690, 2651-2657.

Boorman, P. M., Moynihan, K. J. \& Kerr, K. A. (1981). Chem. Commun. pp. $1286-1288$.

Bruker (2008). APEX2, SAINT-Plus and SADABS. Bruker AXS Inc., Madison, Wisconsin, USA.

Bullock, J. P., Bond, A. M., Boeré, R. T., Gietz, T. M., Roemmele, T. L., Seagrave, S. D., Masuda, J. D. \& Parvez, M. (2013). J. Am. Chem. Soc. 135, 11205-11215.

Chalier, F., Berchadsky, Y., Finet, J., Gronchi, G., Marque, S. \& Tordo, P. (1996). J. Phys. Chem. 100, 4323-4330.

Dunbar, K. R. \& Pence, L. E. (1991). Acta Cryst. C47, $23-26$.

Dunbar, K. R. \& Quillevere, A. (1993). Polyhedron, 12, 807-819.

Hagenbach, A. \& Abram, U. (2005). Z. Anorg. Allg. Chem. 631, 2303-2305.

Jiang, C., Blacque, O. \& Berke, H. (2009). Organometallics, 28, 5233-5239.

Junk, P. C. \& Atwood, J. L. (1999). J. Coord. Chem. 46, 505-518.

Macrae, C. F., Edgington, P. R., McCabe, P., Pidcock, E., Shields, G. P., Taylor, R., Towler, M. \& van de Streek, J. (2006). J. Appl. Cryst. 39, 453-457.

Ménard, G., Hatnean, J. A., Cowley, H. J., Lough, A. J., Rawson, J. M. \& Stephan, D. W. (2013). J. Am. Chem. Soc. 135, 6446-6449.

Ménard, G. \& Stephan, D. W. (2010). J. Am. Chem. Soc. 132, 1796-1797.

Murphy, D. M. (2009). EPR (Electron Paramagnetic Resonance) Spectroscopy of Polycrystalline Oxide Systems, in Metal Oxide Catalysis, pp. 1-50. Weinheim: Wiley-VCH Verlag GmbH \& Co.

Pan, X., Chen, X., Li, T., Li, Y. \& Wang, X. (2013). J. Am. Chem. Soc. 135, 3414-3417.

Sasaki, S., Chowdhury, R. \& Yoshifuji, M. (2004). Tetrahedron Lett. 45, $9193-$ 9196.

Sasaki, S., Murakami, F. \& Yoshifuji, M. (2006). Organometallics, 25, 140147.

Sasaki, S., Sutoh, K., Murakami, F. \& Yoshifuji, M. (2002). J. Am. Chem. Soc. 124, 14830-14831.

Sasaki, S. \& Yoshifuji, M. (2007). Curr. Org. Chem. 38, 17-31.

Schäfer, A., Reissmann, M., Schäfer, A., Saak, W., Haase, D. \& Müller, T. (2011). Angew. Chem. Int. Ed. 50, 12636-12638.

Sheldrick, G. M. (2008). Acta Cryst. A64, 112-122.

Sheldrick, G. M. (2013). SHELXL2013. University of Göttingen, Germany.

Spek, A. L. (2009). Acta Cryst. D65, 148-155.

Westrip, S. P. (2010). J. Appl. Cryst. 43, 920-925. 


\section{supplementary materials}

Acta Cryst. (2013). C69, 1051-1054 [doi:10.1107/S0108270113020647]

\section{A study of the pyramidality index in tris(2,4,6-triisopropylphenyl)phosphonium perchlorate}

\section{René T. Boeré and Yuankui Zhang}

\section{Computing details}

Data collection: APEX2 (Bruker, 2008); cell refinement: SAINT-Plus (Bruker, 2008); data reduction: SAINT-Plus (Bruker, 2008); program(s) used to solve structure: SHELXS97 (Sheldrick, 2008); program(s) used to refine structure:

SHELXL2013 (Sheldrick, 2013); molecular graphics: Mercury (Macrae et al., 2006); software used to prepare material for publication: publCIF (Westrip, 2010).

\section{Tris(2,4,6-triisopropylphenyl)phosphonium perchlorate}

\section{Crystal data}

$\mathrm{C}_{45} \mathrm{H}_{70} \mathrm{P}^{+} \cdot \mathrm{ClO}_{4}{ }^{-}$

$M_{r}=741.43$

Orthorhombic, $P 2_{1} 2_{1} 2_{1}$

$a=13.6822(14) \AA$

$b=17.0621(18) \AA$

$c=18.660(2) \AA$

$V=4356.1(8) \AA^{3}$

$Z=4$

$F(000)=1616$

Data collection

Bruker APEXII CCD area-detector diffractometer

Radiation source: fine-focus sealed tube, Bruker D8

Graphite monochromator

Detector resolution: 66.06 pixels $\mathrm{mm}^{-1}$

phi and $\omega$ scans

Absorption correction: multi-scan

(SADABS; Bruker, 2008)

Refinement

Refinement on $F^{2}$

Least-squares matrix: full

$R\left[F^{2}>2 \sigma\left(F^{2}\right)\right]=0.039$

$w R\left(F^{2}\right)=0.097$

$S=1.02$

10021 reflections

483 parameters

0 restraints

Primary atom site location: structure-invariant direct methods
$D_{\mathrm{x}}=1.131 \mathrm{Mg} \mathrm{m}^{-3}$

Mo $K \alpha$ radiation, $\lambda=0.71073 \AA$

Cell parameters from 9973 reflections

$\theta=2.2-27.4^{\circ}$

$\mu=0.16 \mathrm{~mm}^{-1}$

$T=173 \mathrm{~K}$

Block, red

$0.38 \times 0.21 \times 0.17 \mathrm{~mm}$

$T_{\min }=0.941, T_{\max }=0.973$

61837 measured reflections

10021 independent reflections

7663 reflections with $I>2 \sigma(I)$

$R_{\text {int }}=0.054$

$\theta_{\max }=27.5^{\circ}, \theta_{\min }=1.9^{\circ}$

$h=-17 \rightarrow 17$

$k=-22 \rightarrow 22$

$l=-24 \rightarrow 24$

Secondary atom site location: difference Fourier map

Hydrogen site location: inferred from

neighbouring sites

$\mathrm{H}$ atoms treated by a mixture of independent

and constrained refinement

$w=1 /\left[\sigma^{2}\left(F_{\mathrm{o}}^{2}\right)+(0.0506 P)^{2}\right]$

where $P=\left(F_{\mathrm{o}}{ }^{2}+2 F_{\mathrm{c}}{ }^{2}\right) / 3$

$(\Delta / \sigma)_{\max }<0.001$

$\Delta \rho_{\max }=0.35$ e $\AA^{-3}$ 
$\Delta \rho_{\min }=-0.31$ e $\AA^{-3}$
Absolute structure: Refined as an inversion twin.

Absolute structure parameter: 0.49 (7)

Special details

Experimental. A crystal coated in Paratone (TM) oil was mounted on the end of a thin glass capillary and cooled in the gas stream of the diffractometer Kryoflex device.

Geometry. All e.s.d.'s (except the e.s.d. in the dihedral angle between two 1.s. planes) are estimated using the full covariance matrix. The cell e.s.d.'s are taken into account individually in the estimation of e.s.d.'s in distances, angles and torsion angles; correlations between e.s.d.'s in cell parameters are only used when they are defined by crystal symmetry. An approximate (isotropic) treatment of cell e.s.d.'s is used for estimating e.s.d.'s involving 1.s. planes.

Refinement. Refined as a 2-component inversion twin.

Fractional atomic coordinates and isotropic or equivalent isotropic displacement parameters $\left(\AA^{2}\right)$

\begin{tabular}{|c|c|c|c|c|}
\hline & $x$ & $y$ & $z$ & $U_{\text {iso }} * / U_{\text {eq }}$ \\
\hline $\mathrm{P} 1$ & $0.71447(5)$ & $0.49036(4)$ & $0.96924(3)$ & $0.02084(14)$ \\
\hline H1 & $0.7281(19)$ & $0.4174(15)$ & $0.9396(13)$ & $0.029(7)^{*}$ \\
\hline $\mathrm{C} 1$ & $0.63959(17)$ & $0.53394(15)$ & $0.90014(12)$ & $0.0195(5)$ \\
\hline $\mathrm{C} 2$ & $0.56611(18)$ & $0.48513(15)$ & $0.87098(12)$ & $0.0234(5)$ \\
\hline C3 & 0.50853 (19) & $0.51520(17)$ & $0.81573(13)$ & $0.0288(6)$ \\
\hline H3 & 0.4585 & 0.4833 & 0.7958 & $0.035 *$ \\
\hline $\mathrm{C} 4$ & $0.5221(2)$ & $0.59044(17)$ & $0.78886(13)$ & $0.0300(6)$ \\
\hline $\mathrm{C} 5$ & $0.5947(2)$ & $0.63629(16)$ & $0.81843(13)$ & $0.0294(6)$ \\
\hline H5 & 0.6048 & 0.6873 & 0.7995 & $0.035^{*}$ \\
\hline C6 & $0.65371(18)$ & $0.61146(15)$ & $0.87468(13)$ & $0.0236(5)$ \\
\hline $\mathrm{C} 7$ & 0.54422 (19) & $0.40214(16)$ & $0.89658(14)$ & $0.0283(6)$ \\
\hline H7 & 0.5865 & 0.3920 & 0.9393 & $0.034^{*}$ \\
\hline $\mathrm{C} 8$ & $0.4378(2)$ & $0.3928(2)$ & 0.92063 (19) & $0.0512(9)$ \\
\hline H8A & 0.4277 & 0.3398 & 0.9393 & $0.077^{*}$ \\
\hline H8B & 0.3943 & 0.4017 & 0.8797 & $0.077 *$ \\
\hline $\mathrm{H} 8 \mathrm{C}$ & 0.4234 & 0.4311 & 0.9583 & $0.077 *$ \\
\hline C9 & 0.5711 & 0.34194 (19) & $0.83992(17)$ & $0.0527(9)$ \\
\hline H9A & 0.6397 & 0.3487 & 0.8262 & $0.079 *$ \\
\hline H9B & 0.5294 & 0.3492 & 0.7977 & $0.079^{*}$ \\
\hline $\mathrm{H} 9 \mathrm{C}$ & 0.5615 & 0.2891 & 0.8593 & $0.079 *$ \\
\hline $\mathrm{C} 10$ & $0.7262(2)$ & $0.66905(16)$ & $0.90601(14)$ & $0.0275(6)$ \\
\hline H10 & 0.7506 & 0.6465 & 0.9521 & $0.033^{*}$ \\
\hline $\mathrm{C} 11$ & $0.6792(2)$ & $0.74829(16)$ & $0.92315(16)$ & $0.0374(7)$ \\
\hline H11A & 0.6207 & 0.7401 & 0.9525 & $0.056^{*}$ \\
\hline H11B & 0.6610 & 0.7746 & 0.8784 & $0.056^{*}$ \\
\hline $\mathrm{H} 11 \mathrm{C}$ & 0.7259 & 0.7809 & 0.9495 & $0.056^{*}$ \\
\hline C12 & $0.8143(2)$ & 0.68025 (19) & $0.85671(17)$ & $0.0424(8)$ \\
\hline H12A & 0.7923 & 0.7008 & 0.8104 & $0.064 *$ \\
\hline H12B & 0.8470 & 0.6298 & 0.8495 & $0.064 *$ \\
\hline $\mathrm{H} 12 \mathrm{C}$ & 0.8601 & 0.7174 & 0.8787 & $0.064^{*}$ \\
\hline C13 & $0.4545(2)$ & $0.62553(19)$ & $0.73408(15)$ & $0.0405(7)$ \\
\hline H13 & 0.4855 & 0.6750 & 0.7166 & $0.049 *$ \\
\hline $\mathrm{C} 14$ & $0.3592(3)$ & $0.6481(3)$ & 0.77047 (19) & $0.0691(12)$ \\
\hline H14A & 0.3733 & 0.6806 & 0.8125 & $0.104 *$ \\
\hline
\end{tabular}




\begin{tabular}{|c|c|c|c|c|}
\hline H14B & 0.3246 & 0.6006 & 0.7855 & $0.104 *$ \\
\hline $\mathrm{H} 14 \mathrm{C}$ & 0.3184 & 0.6777 & 0.7368 & $0.104^{*}$ \\
\hline $\mathrm{C} 15$ & $0.4353(3)$ & $0.5749(2)$ & $0.66933(17)$ & $0.0682(13)$ \\
\hline $\mathrm{H} 15 \mathrm{~A}$ & 0.3998 & 0.5276 & 0.6840 & $0.102 *$ \\
\hline H15B & 0.4976 & 0.5599 & 0.6474 & $0.102^{*}$ \\
\hline H15C & 0.3960 & 0.6042 & 0.6346 & $0.102 *$ \\
\hline $\mathrm{C} 16$ & $0.84116(17)$ & $0.51992(14)$ & $0.97472(13)$ & $0.0207(5)$ \\
\hline $\mathrm{C} 17$ & $0.88062(17)$ & $0.56252(14)$ & $1.03215(13)$ & $0.0217(5)$ \\
\hline $\mathrm{C} 18$ & $0.98159(18)$ & $0.57168(15)$ & $1.03514(14)$ & $0.0258(6)$ \\
\hline H18 & 1.0092 & 0.5996 & 1.0742 & $0.031^{*}$ \\
\hline C19 & $1.04335(18)$ & $0.54141(16)$ & $0.98298(14)$ & $0.0265(6)$ \\
\hline $\mathrm{C} 20$ & $1.00227(18)$ & $0.50219(16)$ & $0.92537(14)$ & $0.0282(6)$ \\
\hline $\mathrm{H} 20$ & 1.0440 & 0.4824 & 0.8889 & $0.034^{*}$ \\
\hline $\mathrm{C} 21$ & $0.90236(18)$ & $0.49091(16)$ & $0.91918(13)$ & $0.0249(5)$ \\
\hline $\mathrm{C} 22$ & 0.81955 (18) & $0.60123(15)$ & $1.08964(13)$ & $0.0256(6)$ \\
\hline H22 & 0.7495 & 0.5945 & 1.0758 & $0.031^{*}$ \\
\hline $\mathrm{C} 23$ & $0.8396(2)$ & $0.68941(17)$ & $1.09352(18)$ & $0.0423(8)$ \\
\hline $\mathrm{H} 23 \mathrm{~A}$ & 0.8327 & 0.7124 & 1.0457 & $0.063^{*}$ \\
\hline $\mathrm{H} 23 \mathrm{~B}$ & 0.9062 & 0.6982 & 1.1111 & $0.063^{*}$ \\
\hline $\mathrm{H} 23 \mathrm{C}$ & 0.7928 & 0.7140 & 1.1263 & $0.063 *$ \\
\hline C24 & $0.8328(2)$ & $0.5626(2)$ & $1.16243(14)$ & $0.0424(8)$ \\
\hline $\mathrm{H} 24 \mathrm{~A}$ & 0.9015 & 0.5662 & 1.1769 & $0.064^{*}$ \\
\hline $\mathrm{H} 24 \mathrm{~B}$ & 0.8135 & 0.5074 & 1.1594 & $0.064^{*}$ \\
\hline $\mathrm{H} 24 \mathrm{C}$ & 0.7919 & 0.5894 & 1.1979 & $0.064^{*}$ \\
\hline $\mathrm{C} 25$ & $0.8642(2)$ & $0.44669(17)$ & $0.85350(15)$ & $0.0314(7)$ \\
\hline $\mathrm{H} 25$ & 0.7921 & 0.4558 & 0.8503 & $0.038^{*}$ \\
\hline $\mathrm{C} 26$ & $0.9106(2)$ & $0.4753(2)$ & $0.78344(15)$ & $0.0468(8)$ \\
\hline $\mathrm{H} 26 \mathrm{~A}$ & 0.9800 & 0.4612 & 0.7828 & $0.070^{*}$ \\
\hline H26B & 0.9040 & 0.5324 & 0.7801 & $0.070^{*}$ \\
\hline $\mathrm{H} 26 \mathrm{C}$ & 0.8774 & 0.4508 & 0.7427 & $0.070^{*}$ \\
\hline $\mathrm{C} 27$ & $0.8810(3)$ & 0.35937 (19) & $0.86237(19)$ & $0.0508(9)$ \\
\hline $\mathrm{H} 27 \mathrm{~A}$ & 0.8545 & 0.3422 & 0.9086 & $0.076^{*}$ \\
\hline H27B & 0.9512 & 0.3483 & 0.8607 & $0.076^{*}$ \\
\hline $\mathrm{H} 27 \mathrm{C}$ & 0.8480 & 0.3311 & 0.8236 & $0.076^{*}$ \\
\hline $\mathrm{C} 28$ & $1.15313(19)$ & $0.55200(18)$ & $0.98844(15)$ & $0.0329(7)$ \\
\hline H28 & 1.1677 & 0.5794 & 1.0346 & $0.039^{*}$ \\
\hline $\mathrm{C} 29$ & 1.1909 (2) & $0.60306(19)$ & $0.92731(16)$ & $0.0414(8)$ \\
\hline $\mathrm{H} 29 \mathrm{~A}$ & 1.1575 & 0.6538 & 0.9283 & $0.062^{*}$ \\
\hline H29B & 1.1781 & 0.5770 & 0.8814 & $0.062^{*}$ \\
\hline $\mathrm{H} 29 \mathrm{C}$ & 1.2614 & 0.6111 & 0.9329 & $0.062 *$ \\
\hline $\mathrm{C} 30$ & $1.2057(2)$ & $0.4737(2)$ & 0.98903 (19) & $0.0517(9)$ \\
\hline $\mathrm{H} 30 \mathrm{~A}$ & 1.1978 & 0.4481 & 0.9424 & $0.078^{*}$ \\
\hline H30B & 1.1779 & 0.4403 & 1.0266 & $0.078^{*}$ \\
\hline $\mathrm{H} 30 \mathrm{C}$ & 1.2754 & 0.4821 & 0.9985 & $0.078^{*}$ \\
\hline $\mathrm{C} 31$ & $0.64563(17)$ & $0.47203(14)$ & $1.05060(12)$ & $0.0201(5)$ \\
\hline $\mathrm{C} 32$ & $0.66011(18)$ & $0.39765(15)$ & $1.08289(12)$ & $0.0225(5)$ \\
\hline $\mathrm{C} 33$ & $0.58916(19)$ & $0.37086(15)$ & $1.13016(13)$ & $0.0256(6)$ \\
\hline H33 & 0.5978 & 0.3213 & 1.1524 & $0.031 *$ \\
\hline C34 & 0.50577 (19) & $0.41436(15)$ & $1.14596(13)$ & $0.0246(6)$ \\
\hline
\end{tabular}




\begin{tabular}{lllll} 
C35 & $0.49834(18)$ & $0.48942(15)$ & $1.11824(13)$ & $0.0230(5)$ \\
H35 & 0.4445 & 0.5211 & 1.1321 & $0.028^{*}$ \\
C36 & $0.56674(17)$ & $0.52000(15)$ & $1.07095(12)$ & $0.0206(5)$ \\
C37 & $0.74783(19)$ & $0.34504(16)$ & $1.06815(14)$ & $0.0292(6)$ \\
H37 & 0.7949 & 0.3749 & 1.0375 & $0.035^{*}$ \\
C38 & $0.8002(2)$ & $0.32274(19)$ & $1.13796(16)$ & $0.0445(8)$ \\
H38A & 0.7586 & 0.2873 & 1.1659 & $0.067^{*}$ \\
H38B & 0.8134 & 0.3702 & 1.1659 & $0.067^{*}$ \\
H38C & 0.8620 & 0.2965 & 1.1266 & $0.067^{*}$ \\
C39 & $0.7175(2)$ & $0.27152(16)$ & $1.02758(17)$ & $0.0416(7)$ \\
H39A & 0.6848 & 0.2864 & 0.9829 & $0.062^{*}$ \\
H39B & 0.6726 & 0.2406 & 1.0572 & $0.062^{*}$ \\
H39C & 0.7756 & 0.2402 & 1.0165 & $0.062^{*}$ \\
C40 & $0.55330(18)$ & $0.60468(15)$ & $1.04719(12)$ & $0.0240(6)$ \\
H40 & 0.6086 & 0.6183 & 1.0144 & $0.029^{*}$ \\
C41 & $0.5575(2)$ & $0.66073(16)$ & $1.11175(14)$ & $0.0298(6)$ \\
H41A & 0.5021 & 0.6502 & 1.1437 & $0.045^{*}$ \\
H41B & 0.5542 & 0.7150 & 1.0948 & $0.045^{*}$ \\
H41C & 0.6188 & 0.6526 & 1.1379 & $0.045^{*}$ \\
C42 & $0.4580(2)$ & $0.61740(17)$ & $1.00626(14)$ & $0.0324(6)$ \\
H42A & 0.4025 & 0.6058 & 1.0377 & $0.049^{*}$ \\
H42B & 0.4561 & 0.5825 & 0.9646 & $0.049^{*}$ \\
H42C & 0.4542 & 0.6720 & 0.9902 & $0.049^{*}$ \\
C43 & $0.4206(2)$ & $0.38079(17)$ & $1.18748(15)$ & $0.0332(6)$ \\
H43 & 0.3853 & 0.4254 & 1.2106 & $0.040^{*}$ \\
C44 & $0.4478(2)$ & $0.32272(18)$ & $1.24584(16)$ & $0.0399(7)$ \\
H44A & 0.4910 & 0.3482 & 1.2806 & $0.060^{*}$ \\
H44B & 0.4815 & 0.2778 & 1.2244 & $0.060^{*}$ \\
H44C & 0.3884 & 0.3047 & 1.2701 & $0.060^{*}$ \\
C45 & $0.3518(2)$ & $0.3441(2)$ & $1.13227(17)$ & $0.0465(8)$ \\
H45A & 0.3874 & 0.3049 & 1.1042 & $0.070^{*}$ \\
H45B & 0.3269 & 0.3850 & 1.1002 & $0.070^{*}$ \\
H45C & 0.2970 & 0.3189 & 1.1570 & $0.070^{*}$ \\
C11 & $0.78075(7)$ & $0.88844(5)$ & $0.75076(5)$ & $0.0552(2)$ \\
O1 & $0.7831(4)$ & $0.96017(17)$ & $0.71385(17)$ & $0.1182(14)$ \\
O2 & $0.6876(2)$ & $0.8538(2)$ & $0.74828(17)$ & $0.0901(10)$ \\
O3 & $0.8475(3)$ & $0.8343(2)$ & $0.7175(2)$ & $0.1257(14)$ \\
O4 & $0.8087(2)$ & $0.8991(2)$ & $0.82266(15)$ & $0.0973(11)$ \\
\hline & & & &
\end{tabular}

Atomic displacement parameters $\left(\AA^{2}\right)$

\begin{tabular}{lllllll}
\hline & $U^{11}$ & $U^{22}$ & $U^{33}$ & $U^{12}$ & $U^{13}$ & $U^{23}$ \\
\hline P1 & $0.0185(3)$ & $0.0253(3)$ & $0.0188(3)$ & $-0.0014(3)$ & $0.0000(3)$ & $0.0019(3)$ \\
C1 & $0.0177(12)$ & $0.0262(14)$ & $0.0145(11)$ & $0.0017(10)$ & $-0.0005(9)$ & $-0.0008(10)$ \\
C2 & $0.0241(13)$ & $0.0294(14)$ & $0.0167(11)$ & $0.0011(12)$ & $0.0016(10)$ & $-0.0035(11)$ \\
C3 & $0.0260(14)$ & $0.0398(16)$ & $0.0207(12)$ & $0.0001(13)$ & $-0.0050(10)$ & $-0.0070(12)$ \\
C4 & $0.0323(15)$ & $0.0381(17)$ & $0.0196(13)$ & $0.0091(13)$ & $-0.0004(11)$ & $-0.0019(12)$ \\
C5 & $0.0365(16)$ & $0.0298(15)$ & $0.0219(13)$ & $0.0042(13)$ & $0.0019(12)$ & $0.0049(11)$ \\
C6 & $0.0245(13)$ & $0.0269(14)$ & $0.0194(12)$ & $0.0034(11)$ & $0.0037(10)$ & $0.0002(11)$ \\
C7 & $0.0310(15)$ & $0.0282(15)$ & $0.0258(13)$ & $-0.0065(12)$ & $-0.0027(11)$ & $-0.0034(11)$
\end{tabular}


supplementary materials

\begin{tabular}{|c|c|c|c|c|c|c|}
\hline $\mathrm{C} 8$ & $0.0360(18)$ & $0.057(2)$ & $0.061(2)$ & $-0.0150(17)$ & $0.0068(16)$ & $0.0041(18)$ \\
\hline C9 & 0.080 & $0.0335(18)$ & $0.0446(19)$ & $-0.0040(18)$ & $0.0115(18)$ & $-0.0086(15)$ \\
\hline $\mathrm{C} 10$ & $0.0315(15)$ & $0.0241(14)$ & $0.0270(13)$ & $-0.0010(12)$ & $-0.0014(12)$ & $0.0046(11)$ \\
\hline $\mathrm{C} 11$ & $0.0480(18)$ & $0.0252(15)$ & $0.0389(16)$ & $-0.0005(14)$ & $0.0010(14)$ & $0.0004(13)$ \\
\hline $\mathrm{C} 12$ & $0.0354(17)$ & $0.0398(18)$ & $0.052(2)$ & $-0.0051(14)$ & $0.0081(14)$ & $0.0047(15)$ \\
\hline $\mathrm{C} 13$ & $0.0431(18)$ & $0.0502(19)$ & $0.0282(15)$ & $0.0111(15)$ & $-0.0090(13)$ & $0.0019(14)$ \\
\hline $\mathrm{C} 14$ & $0.063(2)$ & $0.098(3)$ & $0.047(2)$ & $0.051(2)$ & $-0.0156(18)$ & $-0.016(2)$ \\
\hline $\mathrm{C} 15$ & $0.080(3)$ & $0.090(3)$ & $0.0343(18)$ & $0.043(2)$ & $-0.0275(18)$ & $-0.0183(19)$ \\
\hline $\mathrm{C} 16$ & $0.0174(11)$ & $0.0218(13)$ & $0.0228(12)$ & $-0.0001(10)$ & $-0.0003(10)$ & $0.0019(11)$ \\
\hline $\mathrm{C} 17$ & $0.0227(13)$ & $0.0222(13)$ & $0.0201(12)$ & $0.0004(10)$ & $0.0004(10)$ & $0.0000(11)$ \\
\hline $\mathrm{C} 18$ & $0.0216(13)$ & $0.0325(15)$ & $0.0233(12)$ & $-0.0024(11)$ & $-0.0009(11)$ & $-0.0009(12)$ \\
\hline C19 & $0.0192(13)$ & $0.0321(15)$ & $0.0281(14)$ & $0.0016(11)$ & $0.0008(10)$ & $0.0022(12)$ \\
\hline $\mathrm{C} 20$ & $0.0233(13)$ & $0.0339(16)$ & $0.0273(13)$ & $0.0050(12)$ & $0.0048(10)$ & $-0.0025(12)$ \\
\hline $\mathrm{C} 21$ & $0.0254(13)$ & $0.0262(14)$ & $0.0230(12)$ & $-0.0018(11)$ & $0.0020(10)$ & $-0.0019(11)$ \\
\hline $\mathrm{C} 22$ & $0.0190(12)$ & $0.0325(15)$ & $0.0254(13)$ & $-0.0013(11)$ & $0.0016(10)$ & $-0.0082(12)$ \\
\hline $\mathrm{C} 23$ & $0.0353(17)$ & $0.0366(18)$ & $0.055(2)$ & $-0.0014(14)$ & $0.0025(15)$ & $-0.0204(15)$ \\
\hline $\mathrm{C} 24$ & $0.0401(18)$ & $0.063(2)$ & $0.0240(15)$ & $0.0047(16)$ & $0.0042(13)$ & $-0.0059(14)$ \\
\hline $\mathrm{C} 25$ & $0.0240(14)$ & $0.0371(17)$ & $0.0331(15)$ & $-0.0030(12)$ & $0.0032(12)$ & $-0.0133(13)$ \\
\hline $\mathrm{C} 26$ & $0.0512(19)$ & $0.061(2)$ & $0.0283(15)$ & $-0.0086(17)$ & $0.0038(14)$ & $-0.0092(15)$ \\
\hline $\mathrm{C} 27$ & $0.057(2)$ & $0.0396(19)$ & $0.056(2)$ & $-0.0027(16)$ & $0.0104(17)$ & $-0.0166(17)$ \\
\hline $\mathrm{C} 28$ & $0.0186(13)$ & $0.0521(19)$ & $0.0279(15)$ & $-0.0014(13)$ & $-0.0017(11)$ & $-0.0009(13)$ \\
\hline $\mathrm{C} 29$ & $0.0275(16)$ & $0.056(2)$ & $0.0406(16)$ & $-0.0087(15)$ & $0.0012(13)$ & $0.0034(16)$ \\
\hline $\mathrm{C} 30$ & $0.0270(16)$ & $0.065(2)$ & $0.063(2)$ & $0.0111(16)$ & $0.0034(15)$ & $0.0160(18)$ \\
\hline $\mathrm{C} 31$ & $0.0196(12)$ & $0.0250(14)$ & $0.0156(11)$ & $-0.0032(11)$ & $0.0008(9)$ & $0.0026(10)$ \\
\hline $\mathrm{C} 32$ & $0.0243(13)$ & $0.0240(13)$ & $0.0193(12)$ & $0.0012(11)$ & $-0.0014(10)$ & $-0.0018(11)$ \\
\hline $\mathrm{C} 33$ & $0.0298(14)$ & $0.0240(14)$ & $0.0231(13)$ & $-0.0004(11)$ & $0.0011(11)$ & $0.0056(11)$ \\
\hline $\mathrm{C} 34$ & $0.0290(14)$ & $0.0255(14)$ & $0.0194(12)$ & $-0.0034(12)$ & $0.0036(10)$ & $-0.0012(11)$ \\
\hline $\mathrm{C} 35$ & $0.0214(13)$ & $0.0251(14)$ & $0.0226(12)$ & $0.0019(11)$ & $0.0006(10)$ & $-0.0016(11)$ \\
\hline $\mathrm{C} 36$ & $0.0203(12)$ & $0.0244(14)$ & $0.0173(11)$ & $-0.0002(11)$ & $-0.0018(9)$ & $-0.0016(10)$ \\
\hline C37 & $0.0298(15)$ & $0.0280(15)$ & $0.0298(14)$ & $0.0059(12)$ & $0.0059(11)$ & $0.0046(12)$ \\
\hline $\mathrm{C} 38$ & $0.0427(19)$ & $0.0468(19)$ & $0.0439(18)$ & $0.0132(16)$ & $-0.0075(15)$ & $0.0040(15)$ \\
\hline C39 & $0.0512(18)$ & $0.0303(16)$ & $0.0432(16)$ & $0.0136(15)$ & $0.0068(17)$ & $-0.0055(14)$ \\
\hline $\mathrm{C} 40$ & $0.0250(13)$ & $0.0247(14)$ & $0.0221(13)$ & $0.0026(11)$ & $0.0029(10)$ & $0.0024(11)$ \\
\hline C41 & $0.0328(16)$ & $0.0253(15)$ & $0.0313(14)$ & $-0.0007(12)$ & $0.0029(12)$ & $-0.0027(12)$ \\
\hline $\mathrm{C} 42$ & $0.0311(15)$ & $0.0336(16)$ & $0.0325(14)$ & $0.0075(13)$ & $-0.0027(12)$ & $0.0017(12)$ \\
\hline $\mathrm{C} 43$ & $0.0329(16)$ & $0.0317(16)$ & $0.0349(15)$ & $-0.0021(13)$ & $0.0144(12)$ & $0.0037(13)$ \\
\hline $\mathrm{C} 44$ & $0.0462(18)$ & $0.0438(18)$ & $0.0296(15)$ & $-0.0114(14)$ & $0.0043(14)$ & $0.0090(14)$ \\
\hline $\mathrm{C} 45$ & $0.0308(16)$ & $0.061(2)$ & $0.0475(19)$ & $-0.0125(16)$ & $-0.0042(14)$ & $0.0205(17)$ \\
\hline Cl1 & $0.0641(6)$ & $0.0443(5)$ & $0.0572(5)$ & $-0.0157(5)$ & $-0.0263(5)$ & $0.0151(4)$ \\
\hline $\mathrm{O} 1$ & $0.219(4)$ & $0.0507(17)$ & $0.085(2)$ & $-0.015(2)$ & $-0.022(3)$ & $0.0322(16)$ \\
\hline $\mathrm{O} 2$ & $0.0644(19)$ & $0.120(3)$ & $0.086(2)$ & $-0.0278(17)$ & $-0.0308(16)$ & $-0.002(2)$ \\
\hline $\mathrm{O} 3$ & $0.106(3)$ & $0.090(2)$ & $0.182(4)$ & $0.021(2)$ & $0.026(3)$ & $-0.016(3)$ \\
\hline $\mathrm{O} 4$ & $0.112(3)$ & 0.114 & $0.0653(18)$ & $-0.060(2)$ & $-0.0460(18)$ & $0.0211(17)$ \\
\hline
\end{tabular}

Geometric parameters $\left(\AA,{ }^{\circ}\right)$

\begin{tabular}{llll}
\hline $\mathrm{P} 1-\mathrm{C} 1$ & $1.807(2)$ & $\mathrm{C} 24-\mathrm{H} 24 \mathrm{C}$ & 0.9800 \\
$\mathrm{P} 1-\mathrm{C} 16$ & $1.808(2)$ & $\mathrm{C} 25-\mathrm{C} 27$ & $1.517(4)$ \\
$\mathrm{P} 1-\mathrm{C} 31$ & $1.814(2)$ & $\mathrm{C} 25-\mathrm{C} 26$ & $1.533(4)$ \\
$\mathrm{P} 1-\mathrm{H} 1$ & $1.37(3)$ & $\mathrm{C} 25-\mathrm{H} 25$ & 1.0000
\end{tabular}




\begin{tabular}{|c|c|c|c|}
\hline $\mathrm{C} 1-\mathrm{C} 2$ & $1.414(3)$ & $\mathrm{C} 26-\mathrm{H} 26 \mathrm{~A}$ & 0.9800 \\
\hline $\mathrm{C} 1-\mathrm{C} 6$ & $1.419(4)$ & $\mathrm{C} 26-\mathrm{H} 26 \mathrm{~B}$ & 0.9800 \\
\hline $\mathrm{C} 2-\mathrm{C} 3$ & $1.395(3)$ & $\mathrm{C} 26-\mathrm{H} 26 \mathrm{C}$ & 0.9800 \\
\hline $\mathrm{C} 2-\mathrm{C} 7$ & $1.524(4)$ & $\mathrm{C} 27-\mathrm{H} 27 \mathrm{~A}$ & 0.9800 \\
\hline $\mathrm{C} 3-\mathrm{C} 4$ & $1.391(4)$ & $\mathrm{C} 27-\mathrm{H} 27 \mathrm{~B}$ & 0.9800 \\
\hline $\mathrm{C} 3-\mathrm{H} 3$ & 0.9500 & $\mathrm{C} 27-\mathrm{H} 27 \mathrm{C}$ & 0.9800 \\
\hline $\mathrm{C} 4-\mathrm{C} 5$ & $1.379(4)$ & $\mathrm{C} 28-\mathrm{C} 30$ & $1.517(4)$ \\
\hline $\mathrm{C} 4-\mathrm{C} 13$ & $1.503(4)$ & $\mathrm{C} 28-\mathrm{C} 29$ & $1.525(4)$ \\
\hline $\mathrm{C} 5-\mathrm{C} 6$ & $1.390(3)$ & $\mathrm{C} 28-\mathrm{H} 28$ & 1.0000 \\
\hline $\mathrm{C} 5-\mathrm{H} 5$ & 0.9500 & $\mathrm{C} 29-\mathrm{H} 29 \mathrm{~A}$ & 0.9800 \\
\hline $\mathrm{C} 6-\mathrm{C} 10$ & $1.513(4)$ & $\mathrm{C} 29-\mathrm{H} 29 \mathrm{~B}$ & 0.9800 \\
\hline $\mathrm{C} 7-\mathrm{C} 9$ & $1.519(4)$ & $\mathrm{C} 29-\mathrm{H} 29 \mathrm{C}$ & 0.9800 \\
\hline $\mathrm{C} 7-\mathrm{C} 8$ & $1.532(4)$ & $\mathrm{C} 30-\mathrm{H} 30 \mathrm{~A}$ & 0.9800 \\
\hline $\mathrm{C} 7-\mathrm{H} 7$ & 1.0000 & $\mathrm{C} 30-\mathrm{H} 30 \mathrm{~B}$ & 0.9800 \\
\hline $\mathrm{C} 8-\mathrm{H} 8 \mathrm{~A}$ & 0.9800 & $\mathrm{C} 30-\mathrm{H} 30 \mathrm{C}$ & 0.9800 \\
\hline $\mathrm{C} 8-\mathrm{H} 8 \mathrm{~B}$ & 0.9800 & $\mathrm{C} 31-\mathrm{C} 36$ & $1.407(3)$ \\
\hline $\mathrm{C} 8-\mathrm{H} 8 \mathrm{C}$ & 0.9800 & C31-C32 & $1.419(3)$ \\
\hline $\mathrm{C} 9-\mathrm{H} 9 \mathrm{~A}$ & 0.9800 & $\mathrm{C} 32-\mathrm{C} 33$ & $1.389(3)$ \\
\hline C9-H9B & 0.9800 & $\mathrm{C} 32-\mathrm{C} 37$ & $1.524(3)$ \\
\hline $\mathrm{C} 9-\mathrm{H} 9 \mathrm{C}$ & 0.9800 & $\mathrm{C} 33-\mathrm{C} 34$ & $1.393(4)$ \\
\hline $\mathrm{C} 10-\mathrm{C} 12$ & $1.529(4)$ & $\mathrm{C} 33-\mathrm{H} 33$ & 0.9500 \\
\hline $\mathrm{C} 10-\mathrm{C} 11$ & $1.530(4)$ & $\mathrm{C} 34-\mathrm{C} 35$ & $1.385(4)$ \\
\hline $\mathrm{C} 10-\mathrm{H} 10$ & 1.0000 & $\mathrm{C} 34-\mathrm{C} 43$ & $1.512(4)$ \\
\hline $\mathrm{C} 11-\mathrm{H} 11 \mathrm{~A}$ & 0.9800 & $\mathrm{C} 35-\mathrm{C} 36$ & $1.388(3)$ \\
\hline C11-H11B & 0.9800 & C $35-\mathrm{H} 35$ & 0.9500 \\
\hline $\mathrm{C} 11-\mathrm{H} 11 \mathrm{C}$ & 0.9800 & $\mathrm{C} 36-\mathrm{C} 40$ & $1.522(4)$ \\
\hline $\mathrm{C} 12-\mathrm{H} 12 \mathrm{~A}$ & 0.9800 & $\mathrm{C} 37-\mathrm{C} 39$ & $1.523(4)$ \\
\hline $\mathrm{C} 12-\mathrm{H} 12 \mathrm{~B}$ & 0.9800 & $\mathrm{C} 37-\mathrm{C} 38$ & $1.534(4)$ \\
\hline $\mathrm{C} 12-\mathrm{H} 12 \mathrm{C}$ & 0.9800 & C37-H37 & 1.0000 \\
\hline $\mathrm{C} 13-\mathrm{C} 15$ & $1.508(4)$ & C38-H38A & 0.9800 \\
\hline $\mathrm{C} 13-\mathrm{C} 14$ & $1.519(5)$ & C38-H38B & 0.9800 \\
\hline C13-H13 & 1.0000 & $\mathrm{C} 38-\mathrm{H} 38 \mathrm{C}$ & 0.9800 \\
\hline $\mathrm{C} 14-\mathrm{H} 14 \mathrm{~A}$ & 0.9800 & $\mathrm{C} 39-\mathrm{H} 39 \mathrm{~A}$ & 0.9800 \\
\hline C14-H14B & 0.9800 & C39-H39B & 0.9800 \\
\hline $\mathrm{C} 14-\mathrm{H} 14 \mathrm{C}$ & 0.9800 & $\mathrm{C} 39-\mathrm{H} 39 \mathrm{C}$ & 0.9800 \\
\hline $\mathrm{C} 15-\mathrm{H} 15 \mathrm{~A}$ & 0.9800 & $\mathrm{C} 40-\mathrm{C} 42$ & $1.527(4)$ \\
\hline $\mathrm{C} 15-\mathrm{H} 15 \mathrm{~B}$ & 0.9800 & $\mathrm{C} 40-\mathrm{C} 41$ & $1.539(4)$ \\
\hline $\mathrm{C} 15-\mathrm{H} 15 \mathrm{C}$ & 0.9800 & $\mathrm{C} 40-\mathrm{H} 40$ & 1.0000 \\
\hline $\mathrm{C} 16-\mathrm{C} 17$ & $1.403(3)$ & $\mathrm{C} 41-\mathrm{H} 41 \mathrm{~A}$ & 0.9800 \\
\hline $\mathrm{C} 16-\mathrm{C} 21$ & $1.421(3)$ & $\mathrm{C} 41-\mathrm{H} 41 \mathrm{~B}$ & 0.9800 \\
\hline $\mathrm{C} 17-\mathrm{C} 18$ & $1.391(3)$ & $\mathrm{C} 41-\mathrm{H} 41 \mathrm{C}$ & 0.9800 \\
\hline $\mathrm{C} 17-\mathrm{C} 22$ & $1.512(3)$ & $\mathrm{C} 42-\mathrm{H} 42 \mathrm{~A}$ & 0.9800 \\
\hline $\mathrm{C} 18-\mathrm{C} 19$ & $1.389(4)$ & $\mathrm{C} 42-\mathrm{H} 42 \mathrm{~B}$ & 0.9800 \\
\hline $\mathrm{C} 18-\mathrm{H} 18$ & 0.9500 & $\mathrm{C} 42-\mathrm{H} 42 \mathrm{C}$ & 0.9800 \\
\hline $\mathrm{C} 19-\mathrm{C} 20$ & $1.385(4)$ & $\mathrm{C} 43-\mathrm{C} 44$ & $1.518(4)$ \\
\hline $\mathrm{C} 19-\mathrm{C} 28$ & $1.516(4)$ & $\mathrm{C} 43-\mathrm{C} 45$ & $1.530(4)$ \\
\hline $\mathrm{C} 20-\mathrm{C} 21$ & 1.385 & $\mathrm{C} 43-\mathrm{H} 43$ & 1.0000 \\
\hline $\mathrm{C} 20-\mathrm{H} 20$ & 0.9500 & $\mathrm{C} 44-\mathrm{H} 44 \mathrm{~A}$ & 0.9800 \\
\hline $\mathrm{C} 21-\mathrm{C} 25$ & $1.531(3)$ & $\mathrm{C} 44-\mathrm{H} 44 \mathrm{~B}$ & 0.9800 \\
\hline
\end{tabular}




\begin{tabular}{|c|c|c|c|}
\hline $\mathrm{C} 22-\mathrm{C} 24$ & $1.520(4)$ & $\mathrm{C} 44-\mathrm{H} 44 \mathrm{C}$ & 0.9800 \\
\hline $\mathrm{C} 22-\mathrm{C} 23$ & $1.531(4)$ & $\mathrm{C} 45-\mathrm{H} 45 \mathrm{~A}$ & 0.9800 \\
\hline $\mathrm{C} 22-\mathrm{H} 22$ & 1.0000 & $\mathrm{C} 45-\mathrm{H} 45 \mathrm{~B}$ & 0.9800 \\
\hline $\mathrm{C} 23-\mathrm{H} 23 \mathrm{~A}$ & 0.9800 & $\mathrm{C} 45-\mathrm{H} 45 \mathrm{C}$ & 0.9800 \\
\hline $\mathrm{C} 23-\mathrm{H} 23 \mathrm{~B}$ & 0.9800 & $\mathrm{C} 11-\mathrm{O} 1$ & $1.405(3)$ \\
\hline $\mathrm{C} 23-\mathrm{H} 23 \mathrm{C}$ & 0.9800 & $\mathrm{C} 11-\mathrm{O} 2$ & $1.406(3)$ \\
\hline $\mathrm{C} 24-\mathrm{H} 24 \mathrm{~A}$ & 0.9800 & $\mathrm{Cl1}-\mathrm{O} 4$ & $1.407(3)$ \\
\hline $\mathrm{C} 24-\mathrm{H} 24 \mathrm{~B}$ & 0.9800 & $\mathrm{Cl1}-\mathrm{O} 3$ & $1.439(4)$ \\
\hline $\mathrm{C} 1-\mathrm{P} 1-\mathrm{C} 16$ & $117.98(11)$ & $\mathrm{C} 27-\mathrm{C} 25-\mathrm{C} 21$ & $110.2(3)$ \\
\hline $\mathrm{C} 1-\mathrm{P} 1-\mathrm{C} 31$ & $111.95(11)$ & $\mathrm{C} 27-\mathrm{C} 25-\mathrm{C} 26$ & $110.1(3)$ \\
\hline $\mathrm{C} 16-\mathrm{P} 1-\mathrm{C} 31$ & $119.91(11)$ & $\mathrm{C} 21-\mathrm{C} 25-\mathrm{C} 26$ & $112.6(2)$ \\
\hline $\mathrm{C} 1-\mathrm{P} 1-\mathrm{H} 1$ & $99.3(11)$ & $\mathrm{C} 27-\mathrm{C} 25-\mathrm{H} 25$ & 107.9 \\
\hline $\mathrm{C} 16-\mathrm{P} 1-\mathrm{H} 1$ & $98.4(11)$ & $\mathrm{C} 21-\mathrm{C} 25-\mathrm{H} 25$ & 107.9 \\
\hline $\mathrm{C} 31-\mathrm{P} 1-\mathrm{H} 1$ & $104.5(10)$ & $\mathrm{C} 26-\mathrm{C} 25-\mathrm{H} 25$ & 107.9 \\
\hline $\mathrm{C} 2-\mathrm{C} 1-\mathrm{C} 6$ & $121.1(2)$ & $\mathrm{C} 25-\mathrm{C} 26-\mathrm{H} 26 \mathrm{~A}$ & 109.5 \\
\hline $\mathrm{C} 2-\mathrm{C} 1-\mathrm{P} 1$ & $115.81(19)$ & $\mathrm{C} 25-\mathrm{C} 26-\mathrm{H} 26 \mathrm{~B}$ & 109.5 \\
\hline $\mathrm{C} 6-\mathrm{C} 1-\mathrm{P} 1$ & $123.06(18)$ & $\mathrm{H} 26 \mathrm{~A}-\mathrm{C} 26-\mathrm{H} 26 \mathrm{~B}$ & 109.5 \\
\hline $\mathrm{C} 3-\mathrm{C} 2-\mathrm{C} 1$ & $118.0(2)$ & $\mathrm{C} 25-\mathrm{C} 26-\mathrm{H} 26 \mathrm{C}$ & 109.5 \\
\hline $\mathrm{C} 3-\mathrm{C} 2-\mathrm{C} 7$ & $117.5(2)$ & $\mathrm{H} 26 \mathrm{~A}-\mathrm{C} 26-\mathrm{H} 26 \mathrm{C}$ & 109.5 \\
\hline $\mathrm{C} 1-\mathrm{C} 2-\mathrm{C} 7$ & $124.5(2)$ & $\mathrm{H} 26 \mathrm{~B}-\mathrm{C} 26-\mathrm{H} 26 \mathrm{C}$ & 109.5 \\
\hline $\mathrm{C} 4-\mathrm{C} 3-\mathrm{C} 2$ & $122.0(3)$ & $\mathrm{C} 25-\mathrm{C} 27-\mathrm{H} 27 \mathrm{~A}$ & 109.5 \\
\hline $\mathrm{C} 4-\mathrm{C} 3-\mathrm{H} 3$ & 119.0 & $\mathrm{C} 25-\mathrm{C} 27-\mathrm{H} 27 \mathrm{~B}$ & 109.5 \\
\hline $\mathrm{C} 2-\mathrm{C} 3-\mathrm{H} 3$ & 119.0 & $\mathrm{H} 27 \mathrm{~A}-\mathrm{C} 27-\mathrm{H} 27 \mathrm{~B}$ & 109.5 \\
\hline $\mathrm{C} 5-\mathrm{C} 4-\mathrm{C} 3$ & $118.4(2)$ & $\mathrm{C} 25-\mathrm{C} 27-\mathrm{H} 27 \mathrm{C}$ & 109.5 \\
\hline $\mathrm{C} 5-\mathrm{C} 4-\mathrm{C} 13$ & $119.3(3)$ & $\mathrm{H} 27 \mathrm{~A}-\mathrm{C} 27-\mathrm{H} 27 \mathrm{C}$ & 109.5 \\
\hline $\mathrm{C} 3-\mathrm{C} 4-\mathrm{C} 13$ & $122.0(3)$ & $\mathrm{H} 27 \mathrm{~B}-\mathrm{C} 27-\mathrm{H} 27 \mathrm{C}$ & 109.5 \\
\hline $\mathrm{C} 4-\mathrm{C} 5-\mathrm{C} 6$ & $123.2(3)$ & $\mathrm{C} 19-\mathrm{C} 28-\mathrm{C} 30$ & $111.4(2)$ \\
\hline $\mathrm{C} 4-\mathrm{C} 5-\mathrm{H} 5$ & 118.4 & $\mathrm{C} 19-\mathrm{C} 28-\mathrm{C} 29$ & $110.7(2)$ \\
\hline $\mathrm{C} 6-\mathrm{C} 5-\mathrm{H} 5$ & 118.4 & $\mathrm{C} 30-\mathrm{C} 28-\mathrm{C} 29$ & $110.4(2)$ \\
\hline $\mathrm{C} 5-\mathrm{C} 6-\mathrm{C} 1$ & $117.2(2)$ & $\mathrm{C} 19-\mathrm{C} 28-\mathrm{H} 28$ & 108.1 \\
\hline $\mathrm{C} 5-\mathrm{C} 6-\mathrm{C} 10$ & $118.3(2)$ & $\mathrm{C} 30-\mathrm{C} 28-\mathrm{H} 28$ & 108.1 \\
\hline $\mathrm{C} 1-\mathrm{C} 6-\mathrm{C} 10$ & $124.4(2)$ & $\mathrm{C} 29-\mathrm{C} 28-\mathrm{H} 28$ & 108.1 \\
\hline $\mathrm{C} 9-\mathrm{C} 7-\mathrm{C} 2$ & $111.3(2)$ & $\mathrm{C} 28-\mathrm{C} 29-\mathrm{H} 29 \mathrm{~A}$ & 109.5 \\
\hline $\mathrm{C} 9-\mathrm{C} 7-\mathrm{C} 8$ & $111.3(3)$ & $\mathrm{C} 28-\mathrm{C} 29-\mathrm{H} 29 \mathrm{~B}$ & 109.5 \\
\hline $\mathrm{C} 2-\mathrm{C} 7-\mathrm{C} 8$ & $112.0(2)$ & $\mathrm{H} 29 \mathrm{~A}-\mathrm{C} 29-\mathrm{H} 29 \mathrm{~B}$ & 109.5 \\
\hline $\mathrm{C} 9-\mathrm{C} 7-\mathrm{H} 7$ & 107.3 & $\mathrm{C} 28-\mathrm{C} 29-\mathrm{H} 29 \mathrm{C}$ & 109.5 \\
\hline $\mathrm{C} 2-\mathrm{C} 7-\mathrm{H} 7$ & 107.3 & $\mathrm{H} 29 \mathrm{~A}-\mathrm{C} 29-\mathrm{H} 29 \mathrm{C}$ & 109.5 \\
\hline $\mathrm{C} 8-\mathrm{C} 7-\mathrm{H} 7$ & 107.3 & $\mathrm{H} 29 \mathrm{~B}-\mathrm{C} 29-\mathrm{H} 29 \mathrm{C}$ & 109.5 \\
\hline $\mathrm{C} 7-\mathrm{C} 8-\mathrm{H} 8 \mathrm{~A}$ & 109.5 & $\mathrm{C} 28-\mathrm{C} 30-\mathrm{H} 30 \mathrm{~A}$ & 109.5 \\
\hline $\mathrm{C} 7-\mathrm{C} 8-\mathrm{H} 8 \mathrm{~B}$ & 109.5 & $\mathrm{C} 28-\mathrm{C} 30-\mathrm{H} 30 \mathrm{~B}$ & 109.5 \\
\hline $\mathrm{H} 8 \mathrm{~A}-\mathrm{C} 8-\mathrm{H} 8 \mathrm{~B}$ & 109.5 & $\mathrm{H} 30 \mathrm{~A}-\mathrm{C} 30-\mathrm{H} 30 \mathrm{~B}$ & 109.5 \\
\hline $\mathrm{C} 7-\mathrm{C} 8-\mathrm{H} 8 \mathrm{C}$ & 109.5 & $\mathrm{C} 28-\mathrm{C} 30-\mathrm{H} 30 \mathrm{C}$ & 109.5 \\
\hline $\mathrm{H} 8 \mathrm{~A}-\mathrm{C} 8-\mathrm{H} 8 \mathrm{C}$ & 109.5 & $\mathrm{H} 30 \mathrm{~A}-\mathrm{C} 30-\mathrm{H} 30 \mathrm{C}$ & 109.5 \\
\hline $\mathrm{H} 8 \mathrm{~B}-\mathrm{C} 8-\mathrm{H} 8 \mathrm{C}$ & 109.5 & $\mathrm{H} 30 \mathrm{~B}-\mathrm{C} 30-\mathrm{H} 30 \mathrm{C}$ & 109.5 \\
\hline $\mathrm{C} 7-\mathrm{C} 9-\mathrm{H} 9 \mathrm{~A}$ & 109.5 & $\mathrm{C} 36-\mathrm{C} 31-\mathrm{C} 32$ & $120.9(2)$ \\
\hline $\mathrm{C} 7-\mathrm{C} 9-\mathrm{H} 9 \mathrm{~B}$ & 109.5 & $\mathrm{C} 36-\mathrm{C} 31-\mathrm{P} 1$ & $121.60(18)$ \\
\hline $\mathrm{H} 9 \mathrm{~A}-\mathrm{C} 9-\mathrm{H} 9 \mathrm{~B}$ & 109.5 & $\mathrm{C} 32-\mathrm{C} 31-\mathrm{P} 1$ & $115.93(18)$ \\
\hline $\mathrm{C} 7-\mathrm{C} 9-\mathrm{H} 9 \mathrm{C}$ & 109.5 & $\mathrm{C} 33-\mathrm{C} 32-\mathrm{C} 31$ & $117.8(2)$ \\
\hline
\end{tabular}




\begin{tabular}{|c|c|c|c|}
\hline $\mathrm{H} 9 \mathrm{~A}-\mathrm{C} 9-\mathrm{H} 9 \mathrm{C}$ & 109.5 & $\mathrm{C} 33-\mathrm{C} 32-\mathrm{C} 37$ & $118.1(2)$ \\
\hline $\mathrm{H} 9 \mathrm{~B}-\mathrm{C} 9-\mathrm{H} 9 \mathrm{C}$ & 109.5 & $\mathrm{C} 31-\mathrm{C} 32-\mathrm{C} 37$ & $124.1(2)$ \\
\hline $\mathrm{C} 6-\mathrm{C} 10-\mathrm{C} 12$ & $111.4(2)$ & $\mathrm{C} 32-\mathrm{C} 33-\mathrm{C} 34$ & $122.1(2)$ \\
\hline $\mathrm{C} 6-\mathrm{C} 10-\mathrm{C} 11$ & $112.3(2)$ & $\mathrm{C} 32-\mathrm{C} 33-\mathrm{H} 33$ & 118.9 \\
\hline $\mathrm{C} 12-\mathrm{C} 10-\mathrm{C} 11$ & $110.3(2)$ & $\mathrm{C} 34-\mathrm{C} 33-\mathrm{H} 33$ & 118.9 \\
\hline $\mathrm{C} 6-\mathrm{C} 10-\mathrm{H} 10$ & 107.5 & $\mathrm{C} 35-\mathrm{C} 34-\mathrm{C} 33$ & $118.3(2)$ \\
\hline $\mathrm{C} 12-\mathrm{C} 10-\mathrm{H} 10$ & 107.5 & $\mathrm{C} 35-\mathrm{C} 34-\mathrm{C} 43$ & $119.0(2)$ \\
\hline $\mathrm{C} 11-\mathrm{C} 10-\mathrm{H} 10$ & 107.5 & $\mathrm{C} 33-\mathrm{C} 34-\mathrm{C} 43$ & $122.5(2)$ \\
\hline $\mathrm{C} 10-\mathrm{C} 11-\mathrm{H} 11 \mathrm{~A}$ & 109.5 & $\mathrm{C} 34-\mathrm{C} 35-\mathrm{C} 36$ & $122.4(2)$ \\
\hline $\mathrm{C} 10-\mathrm{C} 11-\mathrm{H} 11 \mathrm{~B}$ & 109.5 & $\mathrm{C} 34-\mathrm{C} 35-\mathrm{H} 35$ & 118.8 \\
\hline $\mathrm{H} 11 \mathrm{~A}-\mathrm{C} 11-\mathrm{H} 11 \mathrm{~B}$ & 109.5 & $\mathrm{C} 36-\mathrm{C} 35-\mathrm{H} 35$ & 118.8 \\
\hline $\mathrm{C} 10-\mathrm{C} 11-\mathrm{H} 11 \mathrm{C}$ & 109.5 & $\mathrm{C} 35-\mathrm{C} 36-\mathrm{C} 31$ & $118.0(2)$ \\
\hline $\mathrm{H} 11 \mathrm{~A}-\mathrm{C} 11-\mathrm{H} 11 \mathrm{C}$ & 109.5 & $\mathrm{C} 35-\mathrm{C} 36-\mathrm{C} 40$ & $117.4(2)$ \\
\hline $\mathrm{H} 11 \mathrm{~B}-\mathrm{C} 11-\mathrm{H} 11 \mathrm{C}$ & 109.5 & $\mathrm{C} 31-\mathrm{C} 36-\mathrm{C} 40$ & $124.5(2)$ \\
\hline $\mathrm{C} 10-\mathrm{C} 12-\mathrm{H} 12 \mathrm{~A}$ & 109.5 & $\mathrm{C} 39-\mathrm{C} 37-\mathrm{C} 32$ & $111.1(2)$ \\
\hline $\mathrm{C} 10-\mathrm{C} 12-\mathrm{H} 12 \mathrm{~B}$ & 109.5 & $\mathrm{C} 39-\mathrm{C} 37-\mathrm{C} 38$ & $110.2(2)$ \\
\hline $\mathrm{H} 12 \mathrm{~A}-\mathrm{C} 12-\mathrm{H} 12 \mathrm{~B}$ & 109.5 & $\mathrm{C} 32-\mathrm{C} 37-\mathrm{C} 38$ & $111.1(2)$ \\
\hline $\mathrm{C} 10-\mathrm{C} 12-\mathrm{H} 12 \mathrm{C}$ & 109.5 & $\mathrm{C} 39-\mathrm{C} 37-\mathrm{H} 37$ & 108.1 \\
\hline $\mathrm{H} 12 \mathrm{~A}-\mathrm{C} 12-\mathrm{H} 12 \mathrm{C}$ & 109.5 & $\mathrm{C} 32-\mathrm{C} 37-\mathrm{H} 37$ & 108.1 \\
\hline $\mathrm{H} 12 \mathrm{~B}-\mathrm{C} 12-\mathrm{H} 12 \mathrm{C}$ & 109.5 & C $38-\mathrm{C} 37-\mathrm{H} 37$ & 108.1 \\
\hline $\mathrm{C} 4-\mathrm{C} 13-\mathrm{C} 15$ & $115.1(3)$ & $\mathrm{C} 37-\mathrm{C} 38-\mathrm{H} 38 \mathrm{~A}$ & 109.5 \\
\hline $\mathrm{C} 4-\mathrm{C} 13-\mathrm{C} 14$ & $109.0(2)$ & $\mathrm{C} 37-\mathrm{C} 38-\mathrm{H} 38 \mathrm{~B}$ & 109.5 \\
\hline $\mathrm{C} 15-\mathrm{C} 13-\mathrm{C} 14$ & $110.8(3)$ & $\mathrm{H} 38 \mathrm{~A}-\mathrm{C} 38-\mathrm{H} 38 \mathrm{~B}$ & 109.5 \\
\hline $\mathrm{C} 4-\mathrm{C} 13-\mathrm{H} 13$ & 107.2 & $\mathrm{C} 37-\mathrm{C} 38-\mathrm{H} 38 \mathrm{C}$ & 109.5 \\
\hline $\mathrm{C} 15-\mathrm{C} 13-\mathrm{H} 13$ & 107.2 & $\mathrm{H} 38 \mathrm{~A}-\mathrm{C} 38-\mathrm{H} 38 \mathrm{C}$ & 109.5 \\
\hline $\mathrm{C} 14-\mathrm{C} 13-\mathrm{H} 13$ & 107.2 & $\mathrm{H} 38 \mathrm{~B}-\mathrm{C} 38-\mathrm{H} 38 \mathrm{C}$ & 109.5 \\
\hline $\mathrm{C} 13-\mathrm{C} 14-\mathrm{H} 14 \mathrm{~A}$ & 109.5 & $\mathrm{C} 37-\mathrm{C} 39-\mathrm{H} 39 \mathrm{~A}$ & 109.5 \\
\hline $\mathrm{C} 13-\mathrm{C} 14-\mathrm{H} 14 \mathrm{~B}$ & 109.5 & $\mathrm{C} 37-\mathrm{C} 39-\mathrm{H} 39 \mathrm{~B}$ & 109.5 \\
\hline $\mathrm{H} 14 \mathrm{~A}-\mathrm{C} 14-\mathrm{H} 14 \mathrm{~B}$ & 109.5 & $\mathrm{H} 39 \mathrm{~A}-\mathrm{C} 39-\mathrm{H} 39 \mathrm{~B}$ & 109.5 \\
\hline $\mathrm{C} 13-\mathrm{C} 14-\mathrm{H} 14 \mathrm{C}$ & 109.5 & $\mathrm{C} 37-\mathrm{C} 39-\mathrm{H} 39 \mathrm{C}$ & 109.5 \\
\hline $\mathrm{H} 14 \mathrm{~A}-\mathrm{C} 14-\mathrm{H} 14 \mathrm{C}$ & 109.5 & $\mathrm{H} 39 \mathrm{~A}-\mathrm{C} 39-\mathrm{H} 39 \mathrm{C}$ & 109.5 \\
\hline $\mathrm{H} 14 \mathrm{~B}-\mathrm{C} 14-\mathrm{H} 14 \mathrm{C}$ & 109.5 & $\mathrm{H} 39 \mathrm{~B}-\mathrm{C} 39-\mathrm{H} 39 \mathrm{C}$ & 109.5 \\
\hline $\mathrm{C} 13-\mathrm{C} 15-\mathrm{H} 15 \mathrm{~A}$ & 109.5 & $\mathrm{C} 36-\mathrm{C} 40-\mathrm{C} 42$ & $112.6(2)$ \\
\hline $\mathrm{C} 13-\mathrm{C} 15-\mathrm{H} 15 \mathrm{~B}$ & 109.5 & $\mathrm{C} 36-\mathrm{C} 40-\mathrm{C} 41$ & $110.9(2)$ \\
\hline $\mathrm{H} 15 \mathrm{~A}-\mathrm{C} 15-\mathrm{H} 15 \mathrm{~B}$ & 109.5 & $\mathrm{C} 42-\mathrm{C} 40-\mathrm{C} 41$ & $109.6(2)$ \\
\hline $\mathrm{C} 13-\mathrm{C} 15-\mathrm{H} 15 \mathrm{C}$ & 109.5 & $\mathrm{C} 36-\mathrm{C} 40-\mathrm{H} 40$ & 107.9 \\
\hline $\mathrm{H} 15 \mathrm{~A}-\mathrm{C} 15-\mathrm{H} 15 \mathrm{C}$ & 109.5 & $\mathrm{C} 42-\mathrm{C} 40-\mathrm{H} 40$ & 107.9 \\
\hline $\mathrm{H} 15 \mathrm{~B}-\mathrm{C} 15-\mathrm{H} 15 \mathrm{C}$ & 109.5 & $\mathrm{C} 41-\mathrm{C} 40-\mathrm{H} 40$ & 107.9 \\
\hline $\mathrm{C} 17-\mathrm{C} 16-\mathrm{C} 21$ & $120.7(2)$ & $\mathrm{C} 40-\mathrm{C} 41-\mathrm{H} 41 \mathrm{~A}$ & 109.5 \\
\hline $\mathrm{C} 17-\mathrm{C} 16-\mathrm{P} 1$ & $123.83(18)$ & $\mathrm{C} 40-\mathrm{C} 41-\mathrm{H} 41 \mathrm{~B}$ & 109.5 \\
\hline $\mathrm{C} 21-\mathrm{C} 16-\mathrm{P} 1$ & $115.24(18)$ & $\mathrm{H} 41 \mathrm{~A}-\mathrm{C} 41-\mathrm{H} 41 \mathrm{~B}$ & 109.5 \\
\hline $\mathrm{C} 18-\mathrm{C} 17-\mathrm{C} 16$ & $118.1(2)$ & $\mathrm{C} 40-\mathrm{C} 41-\mathrm{H} 41 \mathrm{C}$ & 109.5 \\
\hline $\mathrm{C} 18-\mathrm{C} 17-\mathrm{C} 22$ & $118.1(2)$ & $\mathrm{H} 41 \mathrm{~A}-\mathrm{C} 41-\mathrm{H} 41 \mathrm{C}$ & 109.5 \\
\hline $\mathrm{C} 16-\mathrm{C} 17-\mathrm{C} 22$ & $123.8(2)$ & $\mathrm{H} 41 \mathrm{~B}-\mathrm{C} 41-\mathrm{H} 41 \mathrm{C}$ & 109.5 \\
\hline $\mathrm{C} 19-\mathrm{C} 18-\mathrm{C} 17$ & $122.3(2)$ & $\mathrm{C} 40-\mathrm{C} 42-\mathrm{H} 42 \mathrm{~A}$ & 109.5 \\
\hline $\mathrm{C} 19-\mathrm{C} 18-\mathrm{H} 18$ & 118.9 & $\mathrm{C} 40-\mathrm{C} 42-\mathrm{H} 42 \mathrm{~B}$ & 109.5 \\
\hline $\mathrm{C} 17-\mathrm{C} 18-\mathrm{H} 18$ & 118.9 & $\mathrm{H} 42 \mathrm{~A}-\mathrm{C} 42-\mathrm{H} 42 \mathrm{~B}$ & 109.5 \\
\hline $\mathrm{C} 20-\mathrm{C} 19-\mathrm{C} 18$ & $118.5(2)$ & $\mathrm{C} 40-\mathrm{C} 42-\mathrm{H} 42 \mathrm{C}$ & 109.5 \\
\hline $\mathrm{C} 20-\mathrm{C} 19-\mathrm{C} 28$ & $120.8(2)$ & $\mathrm{H} 42 \mathrm{~A}-\mathrm{C} 42-\mathrm{H} 42 \mathrm{C}$ & 109.5 \\
\hline
\end{tabular}




\begin{tabular}{|c|c|c|c|}
\hline $\mathrm{C} 18-\mathrm{C} 19-\mathrm{C} 28$ & $120.8(2)$ & $\mathrm{H} 42 \mathrm{~B}-\mathrm{C} 42-\mathrm{H} 42 \mathrm{C}$ & 109.5 \\
\hline $\mathrm{C} 21-\mathrm{C} 20-\mathrm{C} 19$ & $122.2(2)$ & $\mathrm{C} 34-\mathrm{C} 43-\mathrm{C} 44$ & $115.2(2)$ \\
\hline $\mathrm{C} 21-\mathrm{C} 20-\mathrm{H} 20$ & 118.9 & $\mathrm{C} 34-\mathrm{C} 43-\mathrm{C} 45$ & $106.5(2)$ \\
\hline $\mathrm{C} 19-\mathrm{C} 20-\mathrm{H} 20$ & 118.9 & $\mathrm{C} 44-\mathrm{C} 43-\mathrm{C} 45$ & $111.5(2)$ \\
\hline $\mathrm{C} 20-\mathrm{C} 21-\mathrm{C} 16$ & $118.2(2)$ & $\mathrm{C} 34-\mathrm{C} 43-\mathrm{H} 43$ & 107.8 \\
\hline $\mathrm{C} 20-\mathrm{C} 21-\mathrm{C} 25$ & $118.1(2)$ & $\mathrm{C} 44-\mathrm{C} 43-\mathrm{H} 43$ & 107.8 \\
\hline $\mathrm{C} 16-\mathrm{C} 21-\mathrm{C} 25$ & $123.7(2)$ & $\mathrm{C} 45-\mathrm{C} 43-\mathrm{H} 43$ & 107.8 \\
\hline $\mathrm{C} 17-\mathrm{C} 22-\mathrm{C} 24$ & $112.3(2)$ & $\mathrm{C} 43-\mathrm{C} 44-\mathrm{H} 44 \mathrm{~A}$ & 109.5 \\
\hline $\mathrm{C} 17-\mathrm{C} 22-\mathrm{C} 23$ & $111.3(2)$ & $\mathrm{C} 43-\mathrm{C} 44-\mathrm{H} 44 \mathrm{~B}$ & 109.5 \\
\hline $\mathrm{C} 24-\mathrm{C} 22-\mathrm{C} 23$ & $111.2(2)$ & $\mathrm{H} 44 \mathrm{~A}-\mathrm{C} 44-\mathrm{H} 44 \mathrm{~B}$ & 109.5 \\
\hline $\mathrm{C} 17-\mathrm{C} 22-\mathrm{H} 22$ & 107.2 & $\mathrm{C} 43-\mathrm{C} 44-\mathrm{H} 44 \mathrm{C}$ & 109.5 \\
\hline $\mathrm{C} 24-\mathrm{C} 22-\mathrm{H} 22$ & 107.2 & $\mathrm{H} 44 \mathrm{~A}-\mathrm{C} 44-\mathrm{H} 44 \mathrm{C}$ & 109.5 \\
\hline $\mathrm{C} 23-\mathrm{C} 22-\mathrm{H} 22$ & 107.2 & $\mathrm{H} 44 \mathrm{~B}-\mathrm{C} 44-\mathrm{H} 44 \mathrm{C}$ & 109.5 \\
\hline $\mathrm{C} 22-\mathrm{C} 23-\mathrm{H} 23 \mathrm{~A}$ & 109.5 & $\mathrm{C} 43-\mathrm{C} 45-\mathrm{H} 45 \mathrm{~A}$ & 109.5 \\
\hline $\mathrm{C} 22-\mathrm{C} 23-\mathrm{H} 23 \mathrm{~B}$ & 109.5 & $\mathrm{C} 43-\mathrm{C} 45-\mathrm{H} 45 \mathrm{~B}$ & 109.5 \\
\hline $\mathrm{H} 23 \mathrm{~A}-\mathrm{C} 23-\mathrm{H} 23 \mathrm{~B}$ & 109.5 & $\mathrm{H} 45 \mathrm{~A}-\mathrm{C} 45-\mathrm{H} 45 \mathrm{~B}$ & 109.5 \\
\hline $\mathrm{C} 22-\mathrm{C} 23-\mathrm{H} 23 \mathrm{C}$ & 109.5 & $\mathrm{C} 43-\mathrm{C} 45-\mathrm{H} 45 \mathrm{C}$ & 109.5 \\
\hline $\mathrm{H} 23 \mathrm{~A}-\mathrm{C} 23-\mathrm{H} 23 \mathrm{C}$ & 109.5 & $\mathrm{H} 45 \mathrm{~A}-\mathrm{C} 45-\mathrm{H} 45 \mathrm{C}$ & 109.5 \\
\hline $\mathrm{H} 23 \mathrm{~B}-\mathrm{C} 23-\mathrm{H} 23 \mathrm{C}$ & 109.5 & $\mathrm{H} 45 \mathrm{~B}-\mathrm{C} 45-\mathrm{H} 45 \mathrm{C}$ & 109.5 \\
\hline $\mathrm{C} 22-\mathrm{C} 24-\mathrm{H} 24 \mathrm{~A}$ & 109.5 & $\mathrm{O} 1-\mathrm{C} 11-\mathrm{O} 2$ & $111.8(2)$ \\
\hline $\mathrm{C} 22-\mathrm{C} 24-\mathrm{H} 24 \mathrm{~B}$ & 109.5 & $\mathrm{O} 1-\mathrm{Cl1}-\mathrm{O} 4$ & $110.4(2)$ \\
\hline $\mathrm{H} 24 \mathrm{~A}-\mathrm{C} 24-\mathrm{H} 24 \mathrm{~B}$ & 109.5 & $\mathrm{O} 2-\mathrm{Cl1}-\mathrm{O} 4$ & $109.4(2)$ \\
\hline $\mathrm{C} 22-\mathrm{C} 24-\mathrm{H} 24 \mathrm{C}$ & 109.5 & $\mathrm{O} 1-\mathrm{Cl1}-\mathrm{O} 3$ & $109.5(3)$ \\
\hline $\mathrm{H} 24 \mathrm{~A}-\mathrm{C} 24-\mathrm{H} 24 \mathrm{C}$ & 109.5 & $\mathrm{O} 2-\mathrm{C} 11-\mathrm{O} 3$ & $106.9(2)$ \\
\hline $\mathrm{H} 24 \mathrm{~B}-\mathrm{C} 24-\mathrm{H} 24 \mathrm{C}$ & 109.5 & $\mathrm{O} 4-\mathrm{Cl1}-\mathrm{O} 3$ & $108.8(3)$ \\
\hline $\mathrm{C} 16-\mathrm{P} 1-\mathrm{C} 1-\mathrm{C} 2$ & $146.53(17)$ & $\mathrm{C} 17-\mathrm{C} 16-\mathrm{C} 21-\mathrm{C} 20$ & $3.4(4)$ \\
\hline $\mathrm{C} 31-\mathrm{P} 1-\mathrm{C} 1-\mathrm{C} 2$ & $-68.1(2)$ & $\mathrm{P} 1-\mathrm{C} 16-\mathrm{C} 21-\mathrm{C} 20$ & $-171.3(2)$ \\
\hline $\mathrm{C} 16-\mathrm{P} 1-\mathrm{C} 1-\mathrm{C} 6$ & $-32.0(2)$ & $\mathrm{C} 17-\mathrm{C} 16-\mathrm{C} 21-\mathrm{C} 25$ & $-177.6(2)$ \\
\hline $\mathrm{C} 31-\mathrm{P} 1-\mathrm{C} 1-\mathrm{C} 6$ & $113.3(2)$ & $\mathrm{P} 1-\mathrm{C} 16-\mathrm{C} 21-\mathrm{C} 25$ & $7.7(3)$ \\
\hline $\mathrm{C} 6-\mathrm{C} 1-\mathrm{C} 2-\mathrm{C} 3$ & $0.7(3)$ & $\mathrm{C} 18-\mathrm{C} 17-\mathrm{C} 22-\mathrm{C} 24$ & $-69.6(3)$ \\
\hline $\mathrm{P} 1-\mathrm{C} 1-\mathrm{C} 2-\mathrm{C} 3$ & $-177.85(18)$ & $\mathrm{C} 16-\mathrm{C} 17-\mathrm{C} 22-\mathrm{C} 24$ & $112.4(3)$ \\
\hline $\mathrm{C} 6-\mathrm{C} 1-\mathrm{C} 2-\mathrm{C} 7$ & $-178.2(2)$ & $\mathrm{C} 18-\mathrm{C} 17-\mathrm{C} 22-\mathrm{C} 23$ & $55.8(3)$ \\
\hline $\mathrm{P} 1-\mathrm{C} 1-\mathrm{C} 2-\mathrm{C} 7$ & $3.2(3)$ & $\mathrm{C} 16-\mathrm{C} 17-\mathrm{C} 22-\mathrm{C} 23$ & $-122.1(3)$ \\
\hline $\mathrm{C} 1-\mathrm{C} 2-\mathrm{C} 3-\mathrm{C} 4$ & $0.5(4)$ & $\mathrm{C} 20-\mathrm{C} 21-\mathrm{C} 25-\mathrm{C} 27$ & $77.1(3)$ \\
\hline $\mathrm{C} 7-\mathrm{C} 2-\mathrm{C} 3-\mathrm{C} 4$ & $179.5(2)$ & $\mathrm{C} 16-\mathrm{C} 21-\mathrm{C} 25-\mathrm{C} 27$ & $-101.8(3)$ \\
\hline $\mathrm{C} 2-\mathrm{C} 3-\mathrm{C} 4-\mathrm{C} 5$ & $-0.3(4)$ & $\mathrm{C} 20-\mathrm{C} 21-\mathrm{C} 25-\mathrm{C} 26$ & $-46.2(4)$ \\
\hline $\mathrm{C} 2-\mathrm{C} 3-\mathrm{C} 4-\mathrm{C} 13$ & $-174.3(2)$ & $\mathrm{C} 16-\mathrm{C} 21-\mathrm{C} 25-\mathrm{C} 26$ & $134.8(3)$ \\
\hline $\mathrm{C} 3-\mathrm{C} 4-\mathrm{C} 5-\mathrm{C} 6$ & $-1.2(4)$ & $\mathrm{C} 20-\mathrm{C} 19-\mathrm{C} 28-\mathrm{C} 30$ & $-58.8(3)$ \\
\hline $\mathrm{C} 13-\mathrm{C} 4-\mathrm{C} 5-\mathrm{C} 6$ & $173.0(3)$ & $\mathrm{C} 18-\mathrm{C} 19-\mathrm{C} 28-\mathrm{C} 30$ & $122.0(3)$ \\
\hline $\mathrm{C} 4-\mathrm{C} 5-\mathrm{C} 6-\mathrm{C} 1$ & $2.4(4)$ & $\mathrm{C} 20-\mathrm{C} 19-\mathrm{C} 28-\mathrm{C} 29$ & $64.4(3)$ \\
\hline $\mathrm{C} 4-\mathrm{C} 5-\mathrm{C} 6-\mathrm{C} 10$ & $-175.8(2)$ & $\mathrm{C} 18-\mathrm{C} 19-\mathrm{C} 28-\mathrm{C} 29$ & $-114.8(3)$ \\
\hline $\mathrm{C} 2-\mathrm{C} 1-\mathrm{C} 6-\mathrm{C} 5$ & $-2.1(3)$ & $\mathrm{C} 1-\mathrm{P} 1-\mathrm{C} 31-\mathrm{C} 36$ & $-30.9(2)$ \\
\hline $\mathrm{P} 1-\mathrm{C} 1-\mathrm{C} 6-\mathrm{C} 5$ & $176.36(19)$ & $\mathrm{C} 16-\mathrm{P} 1-\mathrm{C} 31-\mathrm{C} 36$ & $113.7(2)$ \\
\hline $\mathrm{C} 2-\mathrm{C} 1-\mathrm{C} 6-\mathrm{C} 10$ & $176.0(2)$ & $\mathrm{C} 1-\mathrm{P} 1-\mathrm{C} 31-\mathrm{C} 32$ & $134.71(18)$ \\
\hline $\mathrm{P} 1-\mathrm{C} 1-\mathrm{C} 6-\mathrm{C} 10$ & $-5.5(3)$ & $\mathrm{C} 16-\mathrm{P} 1-\mathrm{C} 31-\mathrm{C} 32$ & $-80.7(2)$ \\
\hline $\mathrm{C} 3-\mathrm{C} 2-\mathrm{C} 7-\mathrm{C} 9$ & $69.0(3)$ & $\mathrm{C} 36-\mathrm{C} 31-\mathrm{C} 32-\mathrm{C} 33$ & $5.8(3)$ \\
\hline $\mathrm{C} 1-\mathrm{C} 2-\mathrm{C} 7-\mathrm{C} 9$ & $-112.1(3)$ & $\mathrm{P} 1-\mathrm{C} 31-\mathrm{C} 32-\mathrm{C} 33$ & $-159.94(19)$ \\
\hline $\mathrm{C} 3-\mathrm{C} 2-\mathrm{C} 7-\mathrm{C} 8$ & $-56.4(3)$ & $\mathrm{C} 36-\mathrm{C} 31-\mathrm{C} 32-\mathrm{C} 37$ & $-175.0(2)$ \\
\hline
\end{tabular}




\begin{tabular}{llll}
$\mathrm{C} 1-\mathrm{C} 2-\mathrm{C} 7-\mathrm{C} 8$ & $122.6(3)$ & $\mathrm{P} 1-\mathrm{C} 31-\mathrm{C} 32-\mathrm{C} 37$ & $19.2(3)$ \\
$\mathrm{C} 5-\mathrm{C} 6-\mathrm{C} 10-\mathrm{C} 12$ & $-75.3(3)$ & $\mathrm{C} 31-\mathrm{C} 32-\mathrm{C} 33-\mathrm{C} 34$ & $0.3(4)$ \\
$\mathrm{C} 1-\mathrm{C} 6-\mathrm{C} 10-\mathrm{C} 12$ & $106.6(3)$ & $\mathrm{C} 37-\mathrm{C} 32-\mathrm{C} 33-\mathrm{C} 34$ & $-178.9(2)$ \\
$\mathrm{C} 5-\mathrm{C} 6-\mathrm{C} 10-\mathrm{C} 11$ & $48.9(3)$ & $\mathrm{C} 32-\mathrm{C} 33-\mathrm{C} 34-\mathrm{C} 35$ & $-5.7(4)$ \\
$\mathrm{C} 1-\mathrm{C} 6-\mathrm{C} 10-\mathrm{C} 11$ & $-129.2(3)$ & $\mathrm{C} 32-\mathrm{C} 33-\mathrm{C} 34-\mathrm{C} 43$ & $169.8(2)$ \\
$\mathrm{C} 5-\mathrm{C} 4-\mathrm{C} 13-\mathrm{C} 15$ & $135.5(3)$ & $\mathrm{C} 33-\mathrm{C} 34-\mathrm{C} 35-\mathrm{C} 36$ & $5.4(4)$ \\
$\mathrm{C} 3-\mathrm{C} 4-\mathrm{C} 13-\mathrm{C} 15$ & $-50.6(4)$ & $\mathrm{C} 43-\mathrm{C} 34-\mathrm{C} 35-\mathrm{C} 36$ & $-170.3(2)$ \\
$\mathrm{C} 5-\mathrm{C} 4-\mathrm{C} 13-\mathrm{C} 14$ & $-99.5(3)$ & $\mathrm{C} 34-\mathrm{C} 35-\mathrm{C} 36-\mathrm{C} 31$ & $0.5(4)$ \\
$\mathrm{C} 3-\mathrm{C} 4-\mathrm{C} 13-\mathrm{C} 14$ & $74.5(4)$ & $\mathrm{C} 34-\mathrm{C} 35-\mathrm{C} 36-\mathrm{C} 40$ & $-177.0(2)$ \\
$\mathrm{C} 1-\mathrm{P} 1-\mathrm{C} 16-\mathrm{C} 17$ & $113.2(2)$ & $\mathrm{C} 32-\mathrm{C} 31-\mathrm{C} 36-\mathrm{C} 35$ & $-6.2(3)$ \\
$\mathrm{C} 31-\mathrm{P} 1-\mathrm{C} 16-\mathrm{C} 17$ & $-29.3(3)$ & $\mathrm{P} 1-\mathrm{C} 31-\mathrm{C} 36-\mathrm{C} 35$ & $158.77(18)$ \\
$\mathrm{C} 1-\mathrm{P} 1-\mathrm{C} 16-\mathrm{C} 21$ & $-72.3(2)$ & $\mathrm{C} 32-\mathrm{C} 31-\mathrm{C} 36-\mathrm{C} 40$ & $171.1(2)$ \\
$\mathrm{C} 31-\mathrm{P} 1-\mathrm{C} 16-\mathrm{C} 21$ & $145.16(19)$ & $\mathrm{P} 1-\mathrm{C} 31-\mathrm{C} 36-\mathrm{C} 40$ & $-24.0(3)$ \\
$\mathrm{C} 21-\mathrm{C} 16-\mathrm{C} 17-\mathrm{C} 18$ & $-3.5(4)$ & $\mathrm{C} 33-\mathrm{C} 32-\mathrm{C} 37-\mathrm{C} 39$ & $67.4(3)$ \\
$\mathrm{P} 1-\mathrm{C} 16-\mathrm{C} 17-\mathrm{C} 18$ & $170.7(2)$ & $\mathrm{C} 31-\mathrm{C} 32-\mathrm{C} 37-\mathrm{C} 39$ & $-111.7(3)$ \\
$\mathrm{C} 21-\mathrm{C} 16-\mathrm{C} 17-\mathrm{C} 22$ & $174.4(2)$ & $\mathrm{C} 33-\mathrm{C} 32-\mathrm{C} 37-\mathrm{C} 38$ & $-55.6(3)$ \\
$\mathrm{P} 1-\mathrm{C} 16-\mathrm{C} 17-\mathrm{C} 22$ & $-11.3(4)$ & $\mathrm{C} 31-\mathrm{C} 32-\mathrm{C} 37-\mathrm{C} 38$ & $125.2(3)$ \\
$\mathrm{C} 16-\mathrm{C} 17-\mathrm{C} 18-\mathrm{C} 19$ & $1.1(4)$ & $\mathrm{C} 35-\mathrm{C} 36-\mathrm{C} 40-\mathrm{C} 42$ & $-62.3(3)$ \\
$\mathrm{C} 22-\mathrm{C} 17-\mathrm{C} 18-\mathrm{C} 19$ & $-177.0(2)$ & $\mathrm{C} 31-\mathrm{C} 36-\mathrm{C} 40-\mathrm{C} 42$ & $120.5(3)$ \\
$\mathrm{C} 17-\mathrm{C} 18-\mathrm{C} 19-\mathrm{C} 20$ & $1.3(4)$ & $\mathrm{C} 35-\mathrm{C} 36-\mathrm{C} 40-\mathrm{C} 41$ & $60.9(3)$ \\
$\mathrm{C} 17-\mathrm{C} 18-\mathrm{C} 19-\mathrm{C} 28$ & $-179.5(3)$ & $\mathrm{C} 31-\mathrm{C} 36-\mathrm{C} 40-\mathrm{C} 41$ & $-116.4(3)$ \\
$\mathrm{C} 18-\mathrm{C} 19-\mathrm{C} 20-\mathrm{C} 21$ & $-1.4(4)$ & $\mathrm{C} 35-\mathrm{C} 34-\mathrm{C} 43-\mathrm{C} 44$ & $-149.6(3)$ \\
$\mathrm{C} 28-\mathrm{C} 19-\mathrm{C} 20-\mathrm{C} 21$ & $179.4(3)$ & $\mathrm{C} 33-\mathrm{C} 34-\mathrm{C} 43-\mathrm{C} 44$ & $34.9(4)$ \\
$\mathrm{C} 19-\mathrm{C} 20-\mathrm{C} 21-\mathrm{C} 16$ & $-0.9(4)$ & $\mathrm{C} 35-\mathrm{C} 34-\mathrm{C} 43-\mathrm{C} 45$ & $86.2(3)$ \\
$\mathrm{C} 19-\mathrm{C} 20-\mathrm{C} 21-\mathrm{C} 25$ & $-179.9(3)$ & $\mathrm{C} 33-\mathrm{C} 34-\mathrm{C} 43-\mathrm{C} 45$ & $-89.3(3)$ \\
\hline
\end{tabular}

Pyramidality index in triarylphosphonium ion structures

\begin{tabular}{llll}
\hline Aryl group & CSD refcode & $\Sigma(\mathrm{C}-\mathrm{P}-\mathrm{C})\left(^{\circ}\right)$ & Reference \\
$\mathrm{Ph}_{3} \mathrm{PPH}^{+}$ & ABERAA & $332.7(5)$ & Junk \& Atwood (1999) \\
$\mathrm{Ph}_{3} \mathrm{PPH}^{+}$ & BARVUL & $334(3)$ & Boorman et al. $(1981)$ \\
$\mathrm{Ph}_{3} \mathrm{PPH}^{+}$ & KAXQUQ & $333.0(6)$ & Hagenbach \& Abram $(2005)$ \\
$\left(2,4,6-\mathrm{MeOC}_{6} \mathrm{H}_{2}\right)_{3} \mathrm{PPH}^{+}$ & LAFGEE10 & $338.9(5)$ & Dunbar \& Quillevere $(1993)$ \\
$\left(2,4,6-\mathrm{MeOC}_{6} \mathrm{H}_{2}\right)_{3} \mathrm{PPH}^{+}$ & TAJGAM & $342.3(6)$ & Dunbar \& Pence (1991) \\
$\left(2,4,6-\mathrm{MeOC}_{6} \mathrm{H}_{2}\right)_{3} \mathrm{PPH}^{+}$ & WIBJEW & $337.5(9)$ & Dunbar \& Quillevere $(1993)$ \\
$\left(2,4,6-\mathrm{MeOC}_{6} \mathrm{H}_{2}\right)_{3} \mathrm{PPH}^{+}$ & WIBJIA & $343.0(7)$ & Dunbar \& Quillevere (1993) \\
$\mathrm{Mes}_{3} \mathrm{PPH}^{+}$ & LUWPOJ & $345.3(5)$ & Ménard \& Stephan $(2010)$ \\
$\mathrm{Mes}_{3} \mathrm{PPH}^{+}$ & QUHCAY & $345.6(6)$ & Jiang et al. $(2009)$ \\
$\mathrm{Mes}_{3} \mathrm{PPH}^{+}$ & XALCUK & $345.1(6)$ & Schäfer et al. $(2011)$ \\
$\mathrm{Tripp}_{3} \mathrm{PPH}^{+}$ & & $349.9(6)$ & This work \\
\hline
\end{tabular}

